\title{
DNA methylation in colorectal cancer - Impact on screening and therapy monitoring modalities?
}

\author{
Marion Zitt ${ }^{\mathrm{a}, \mathrm{b}}$, Matthias Zitt ${ }^{\mathrm{a}}$ and Hannes M. Müller ${ }^{\mathrm{a}, *}$ \\ ${ }^{a}$ Department of General and Transplant Surgery, Innsbruck Medical University, A-6020 Innsbruck, Austria \\ ${ }^{\mathrm{b}}$ Tyrolean Cancer Research Institute, Innrain 66, A-6020 Innsbruck, Austria
}

\begin{abstract}
Colorectal cancer (CRC) is a common malignancy. It arises from benign neoplasms and evolves into adenocarcinomas through a stepwise histological progression sequence, proceeding from either adenomas or hyperplastic polyps/serrated adenomas. Genetic alterations have been associated with specific steps in this adenoma-carcinoma sequence and are believed to drive the histological progression of CRC. Recently, epigenetic alterations (especially DNA methylation) have been shown to occur in colon polyps and CRC. The aberrant methylation of genes appears to act together with genetic alterations to drive the initiation and progression of colon polyps to CRC.

DNA methylation changes have been recognized as one of the most common molecular alterations in human tumors, including CRC. Because of the ubiquity of DNA methylation changes and the ability to detect methylated DNA in several body fluids (blood, stool), this specifically altered DNA may serve, on the one hand, as a possible new screening marker for CRC and, on the other hand, as a tool for therapy monitoring in patients having had neoplastic disease of the colorectum.

As many CRC patients present with advanced disease, early detection seems to be one of the most important approaches to reduce mortality. Therefore, an effective screening test would have substantial clinical benefits. Furthermore, early detection of progression of disease in patients having had CRC permits immediate commencement of specific treatment regimens (e.g. curative resection of liver and lung metastases) and probably longer survival and better quality of life.
\end{abstract}

Keywords: Colorectal cancer, DNA methylation, stool, screening, therapy monitoring, review

\section{Colorectal cancer in general}

Colorectal cancer (CRC) is the third most common malignant neoplasm worldwide [1]. The annual incidence of CRC in North America and Europe is approximately 30-50/100 000 [2]. In 2005, the American Cancer Society estimated 145290 new cases of and 56290 deaths from CRC in the United States. Thus, $\mathrm{CRC}$ is the third most common malignancy and the third-leading cause of cancer death in women and men in the United States. In women, it ranks third after

*Corresponding author: Hannes M. Müller, MD, Department of General and Transplant Surgery, Innsbruck Medical University, A6020 Innsbruck, Austria. Tel.: +43 512504 22555; Fax: +43 512 504 28519; E-mail: hannes.mueller@uibk.ac.at. lung and breast cancer; in men, it ranks third after lung and prostate cancer [3]. The lifetime incidence of CRC among women and men at average risk is sufficiently high at $6 \%$, or 1 in 18 [4].

Despite advances in surgical techniques and adjuvant therapy, there has been only a modest improvement in survival for patients with advanced neoplasms [5]. Hence, effective primary and secondary preventive approaches must be developed to reduce mortality from CRC. Genetics, experimental and epidemiologic studies suggest that CRC results from complex interactions between inherited susceptibility and environmental factors [6-9].

Because of the frequency of the disease, demonstrated slow growth of primary lesions and better survival of patients with early-stage lesions, CRC is a suit- 
able disease for screening. Several studies have demonstrated benefits of CRC screening [10-12]. Most CRCs appear in the rectum (38\%) or sigmoid colon (29\%) and therefore around two-thirds of CRCs fall within the reach of sigmoidoscopy [13].

\section{Early detection in general}

Advances in cancer treatment and improvements in cancer outcome over the past few decades have been modest, despite significant investment in cancer research. A great deal of research is invested in improving treatment for advanced disease, because most people who develop cancer have advanced disease at the time of diagnosis. For example, of those with lung, colorectal or breast cancer in the United States, $72 \%$, $57 \%$ and $34 \%$, respectively, have regional or distant spread of their disease at the time of diagnosis. Despite huge effort, only modest gains in the survival of cancer patients with advanced disease at the time of diagnosis have been achieved over the past few decades. Comparably less effort has been put into strategies for the early detection of cancer although the promise of early detection is that it will identify cancer while still localized and curable, not only preventing mortality, but also reducing morbidity and costs [14].

Cervical cancer (CC) provides an excellent example of the power of early detection, and subsequent treatment, in reducing the burden of cancer. CC is also an excellent example of intensive research into molecular alterations during pathogenesis of a specific cancer type and subsequently of the establishment of evidence-based screening programs and even therapy or prevention strategies. That is why the history of CC research can serve as an interesting example of how to introduce an efficient screening program and develop new options for therapy or prevention. Therefore, it should be examined more closely:

At the beginning of the twentieth century, mortality due to invasive $\mathrm{CC}$ was among the highest for women. By the middle of the twentieth century, pathologists had shown that the natural history of CC progressed through stages of increasingly severe cervical intraepithelial neoplasia (CIN) and that these stages could be histologically identified using exfoliated cells. Subsequently, an exfoliated cytological staining procedure (Papanicolau (PAP) smear) that can detect premalignant and malignant changes in the cervical epithelium was developed. Furthermore, programes and policies were introduced in developed countries to implement widespread early detection of pre-neoplastic cervical lesions. Since 1950, there has been an approximately $70 \%$ decline in the incidence of, and mortality due to, invasive $\mathrm{CC}$ in the United States, whereas in developing countries where PAP smear screening is not widespread, CC remains a major public health problem [14]. The effectiveness of this screening approach is mostly due to its high acceptance in the population, to the fact that the "organ of interest" is easily accessible in a non-invasive procedure and the fact that changes can easily be identified by a very well established marker like cytology. Detailed knowledge of the alterations during pathogenesis (progressing from low-grade to intermediate- to high-grade CIN and eventually to invasive cancer) has also contributed to the cytological screening of CC.

New technologies - including DNA methylation analyses - offer a variety of new opportunities for developing biomarker-based tests that are less expensive and more accurate than currently used screening tests. Additionally, CC also illustrates the potential power of using molecular tests to enhance the accuracy of early detection. PAP smears are performed on millions of women each year. Thus a large number of both falsenegatives and false-positives occur. The development of molecular methods to augment, or possibly replace, PAP smears has been spurred by the recognition that cervical neoplasia is caused by persistent infection with oncogenic human papillomaviruses (HPVs). Since the late 1990s, studies have shown that relatively inexpensive, easy-to-use, molecular tests for the presence of HPV can be performed on cervical swabs collected either by a practitioner or by a woman herself and will detect pre-invasive $\mathrm{CC}$ with greater sensitivity and no (or slight) loss of specificity in comparison to PAP smears (for review see [14]).

The challenges faced by early-detection researchers can be classified in terms of the steps needed to produce a useful population screening test: discovery, development and evaluation. Sullivan Pepe et al. [15] reported five phases of biomarker development for early detection of cancer: Phase 1 is represented by preclinical exploratory studies aiming to evaluate the expression and regulation of thousands of genes and proteins in tumor and comparable healthy organ tissue to identify candidates for early detection. Phase 2 includes assay development and validation, which is realized with markers in specimens that can be obtained noninvasively, such as serum, plasma, urine, sputum or stool and that correlate with disease. The goal of phase 2 is to evaluate ability of these markers to discrimi- 
nate between patients with clinically established disease and healthy controls. Phase 3 uses retrospective, longitudinal studies. This phase relies on the existence of repositories of clinical specimens, typically serum, that have been routinely collected and stored. Samples obtained from individuals before they were diagnosed with the cancer of interest are compared with samples from healthy age-matched controls. Phase 3 is vitally important because it provides a window on the natural history of the disease and how it relates to levels of the biomarker under study. If more samples are available during the prediagnostic period of the cancer patients, phase 3 studies can determine how long before normal clinical diagnosis a tumor marker might be able to detect disease. Phase 4 uses prospective screening studies to evaluate whether the potential screening marker is, in fact, able to detect the disease while it is still localized, and to estimate expected screening costs. Phase 5 includes cancer control studies which should directly evaluate the impact of screening on population disease morbidity and mortality. Although the five phases are not necessarily sequential, they are ordered according to strength of evidence from weakest to strongest, and results from earlier phases will typically be required to justify conducting later-phase studies $[14,15]$.

Furthermore, it must also be mentioned that the power of a screening test is not only dependent on its specificity and sensitivity, but also on people's willingness to participate in a given screening program. This willingness is strongly influenced by whether the screening is easy to perform, safe and practicable in clinical routine. For example, it was recently reported by the American Cancer Society [12] that $88 \%$ of American women aged between 18 and 44 undergo PAP smear testing, which is an easy-to-perform and safe screening tool. In comparison, $60.5 \%$ of American women aged 40 to 64 undergo mammogram examination, which needs technical equipment and can be painful. Additionally, about 50\% of American men were screened by digital rectal examination and PSA testing for prostate cancer. This very low number may reflect the reduced willingness of men to consult a doctor for screening purposes. With regard to CRC screening, only $40 \%$ of American women and men aged 50 yrs and older (without any sex-specific differences in the prevalence of screening) reported recent screening with an endoscopic procedure (either sigmoidoscopy or colonoscopy) and only $20 \%$ of either sex reported having undergone screening with a fecal occult blood test (FOBT). The reasons for such a low number of participants may be uncomfortable and unpleasant prepara- tion procedures for endoscopy, sometimes painful examination procedures, complications during endoscopy and low sensitivity or specificity of FOBT. Because the prevalence of CRC screening is only approximately $50 \%$ (FOBT or lower endoscopy, or both) the substantial problem of too many average risk adults not being screened with any of the recommended tests persists [12].

\section{Established screening modalities for colorectal cancer}

Few of the advantages of CC screening are true for CRC. Despite its negative aspects, a huge effort to improve CRC screening has been made. CRC is a suitable disease for screening as it has a recognizable early stage and a defined natural history (with a long asymptomatic preclinical phase), surgical treatment is effective, and benefit is greater in early-stage disease.

In an interesting study, Hamilton et al. [16] evaluated the prediagnostic features of CRC using a populationbased case-control study. In total, ten features were statistically significantly associated with CRC before diagnosis: rectal bleeding, weight loss, abdominal pain, diarrhea, constipation, abnormal rectal examination, abdominal tenderness, hemoglobin $<10.0 \mathrm{~g} / \mathrm{dl}$, positive FOBT, blood glucose $>10 \mathrm{mM} / \mathrm{l}$. Furthermore, five of these features (abdominal pain, rectal bleeding, anemia, positive FOBT, raised blood glucose) remained statistically significantly associated with CRC for 180 days before diagnosis [16]. As a consequence, it should be kept in mind that people with symptoms or signs that suggest the presence of CRC or polyps fall outside the domain of screening and should be offered an appropriate diagnostic evaluation for earlier diagnosis of CRC.

Screening programs should begin by classifying the individual patient's level of risk based on personal (CRC or an adenomatous polyp in the patient), family (CRC or an adenomatous polyp in patient's family) and medical history (e.g., inflammatory bowel disease), which will determine the appropriate approach to screening in that person. Clinicians should determine an individual patient's risk status well before the earliest potential initiation of screening (typically around age 20 years, but earlier if there is a family history of familial adenomatous polyposis (FAP)), and any predisposition to CRC should prompt further efforts to identify and define the specific condition associated with increased risk (for review see [10]). Men and women at 
Table 1

Comparison of screening tests for colorectal cancer

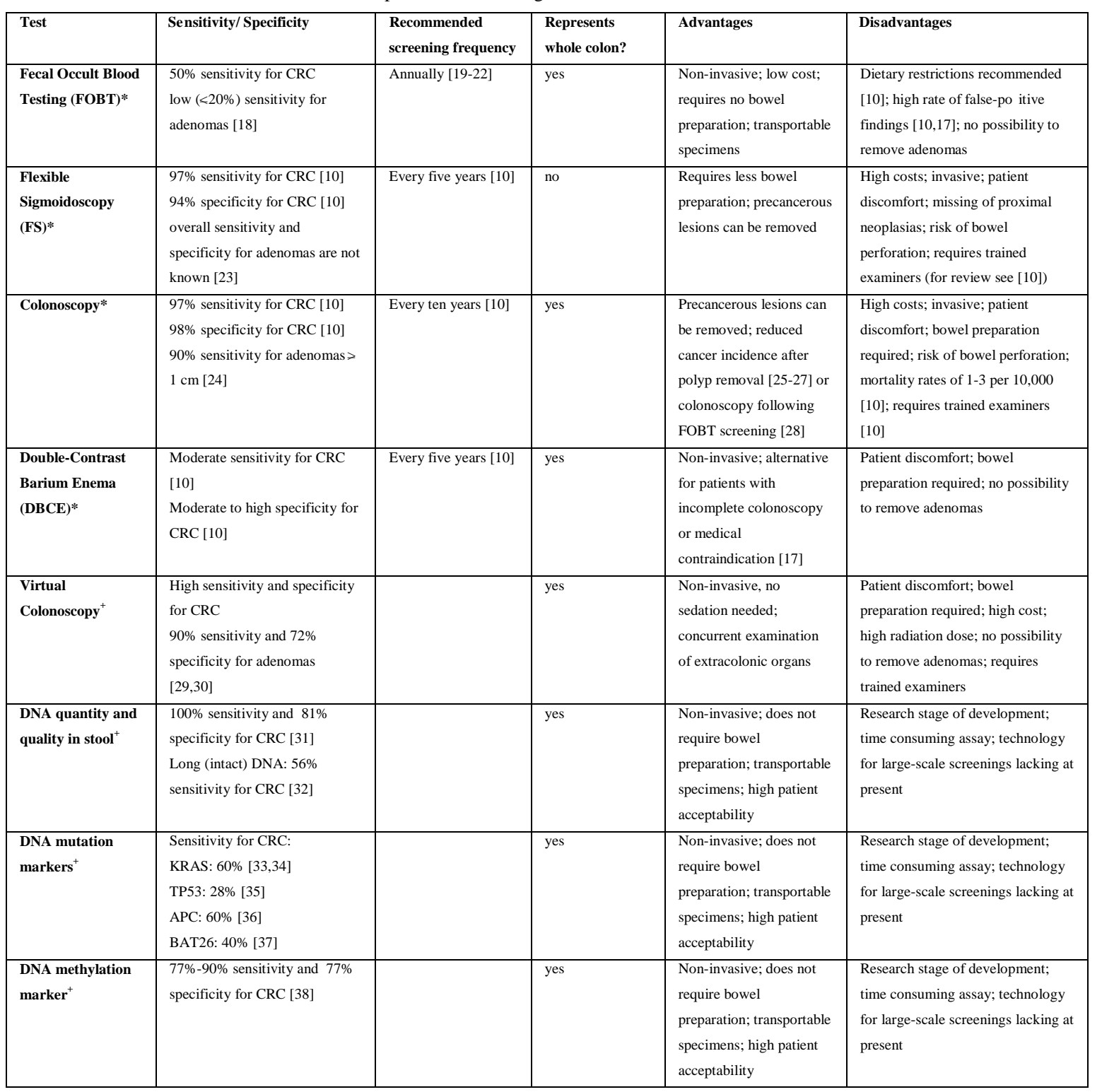

*Established Screening Tests. ${ }^{+}$Emerging Screening Tests

average risk should be offered screening for CRC and adenomatous polyps beginning at the age of 50 years. If the result of a screening test is abnormal, physicians should recommend a complete structural examination of the colon and rectum by colonoscopy (or flexible sigmoidoscopy and double-contrast barium enema if colonoscopy is not available). Screening strategies are not equal with regard to evidence of effectiveness, magnitude of effectiveness, risk, or up-front costs (for review see $[10,17])$. A comparison of potential screening tests for colorectal cancer is presented in Table 1.

\section{Follow-up after curative resection for CRC}

At diagnosis most patients undergo curative resection if CRC is limited to the bowel and the regional lymph nodes. Nevertheless, $30 \%-50 \%$ of patients will have recurrent disease and die of metastatic CRC despite initial radical resection $[39,40]$. In asymptomatic 
patients, detection of a recurrent or metachronous tumor at an early stage improves the chance of another curative resection and may thereby increase overall patient survival. In particular, local recurrences as well as liver and lung metastases may not be clinically symptomatic when diagnosed during follow-up. Since CRC is one of the few solid tumors that may still be surgically treated in curative intention when recurrent disease has been diagnosed [41-43], different strategies to follow up patients after surgery have been developed ([44] and see CRC surveillance guidelines [45]). Additionally, it was seen that the practice of including patients with CRC in surveillance protocols for intensive follow-up significantly improved survival [46].

CRC recurrent disease mainly occurs in the regional abdominal lymph nodes, the liver or the lung and less frequently in the resected segment of the colorectum. Nevertheless, the life-threatening event in CRC is not lymph node metastasis per se, but hematogenous metastases which mainly affect the liver or the lung. Therefore, a screening test that is sensitive for hematogenous metastases and can be performed in patients' serum or plasma may have an impact on early detection of disease progression in patients having had CRC (Fig. 1). Such a test subsequently permits immediate start of specific treatment regimens and probably longer survival and better quality of life.

An increasing number of studies have reported the presence of methylated DNA in serum/plasma of patients with various types of malignancies and the absence of methylated DNA in normal control patients (for review see [47]). For the past five years our research group was mainly interested in evaluating DNA methylation changes in serum of cancer patients [4752]. The potential for using epigenetic markers for prognostication and even for therapy monitoring in CRC appears to be great. A summary of reported methylated genes in serum/plasma of CRC patients is presented in Table 2c.

\section{Adenoma-carcinoma sequence - genetic and epigenetic alterations}

CRC develops as a result of the progressive accumulation of genetic and epigenetic alterations that lead up to the transformation of normal colonic epithelium to colon adenocarcinoma. The fact that CRC develops over about 5-15 years and progresses through parallel histological and molecular changes has permitted a detailed analysis of the events involved in its initiation and progression: firstly, cancer emerges via a multistep progression at both the molecular and the morphological level [7]; secondly, genetic and epigenetic alterations are pathogenic key events in cancer formation driving the initiation and progression of the adenomacarcinoma sequence [53]; thirdly, it has been seen that hereditary cancer syndromes frequently correspond to germline forms of genetic and epigenetic key defects, whose somatic occurrences drive the emergence of sporadic CRC [54]. CRC is most commonly initiated by aberrant accumulation of beta-catenin in the Wingless/Wnt signaling pathway leading to transcription of WNT-target genes. Furthermore, other alterations that have been shown to play a central role in colorectal carcinogenesis affect KRAS2, TP53 and elements of the TGF (transforming growth factor)-b signaling pathway, such as TGFBR2 and MADH4/SMAD4. Epigenetic alterations - particularly aberrant DNA methylation appear to affect genes whose inactivation can promote tumor formation by creating genomic instability (e.g. $M L H 1$ (mutL homologue 1)) or by causing primary inactivation of the methylated gene itself (e.g. $C D K N 2 A$ ) (for review see [55]).

\subsection{Adenoma-carcinoma sequence - genetic alterations}

Most sporadic CRCs are thought to develop from benign adenomas. Identification of the genetic abnormalities that seem to accumulate in a stepwise manner has led to the well-known model of the adenoma-carcinoma sequence $[7,54,56]$. The earliest identifiable lesion in CRC formation appears to be the ACF (aberrant crypt focus). The true neoplastic potential of this lesion is still undetermined, but it has been shown that some of these lesions harbor mutations in KRAS2 or APC (adenomatous polyposis coli) and can progress to CRC (for review see [55]).

Accumulating evidence demonstrates that some CRC arise from hyperplastic polyps via a serrated adenoma intermediate [57]. Interestingly, this hyperplastic polyp-serrated adenoma-adenocarcinoma sequence is more common in the proximal colon, and these tumors more often show increased DNA methylation and mutations in BRAF [58,59].

Adenomas are generally masses that protrude into the gut lumen (polyps). They can either be pedunculated (with a stalk) or sessile (without a stalk). More rarely, adenomas can be flat or depressed. The epithelium of adenomas can form glands (tubular adenoma), finger-like projections (villous adenoma) or a combi- 


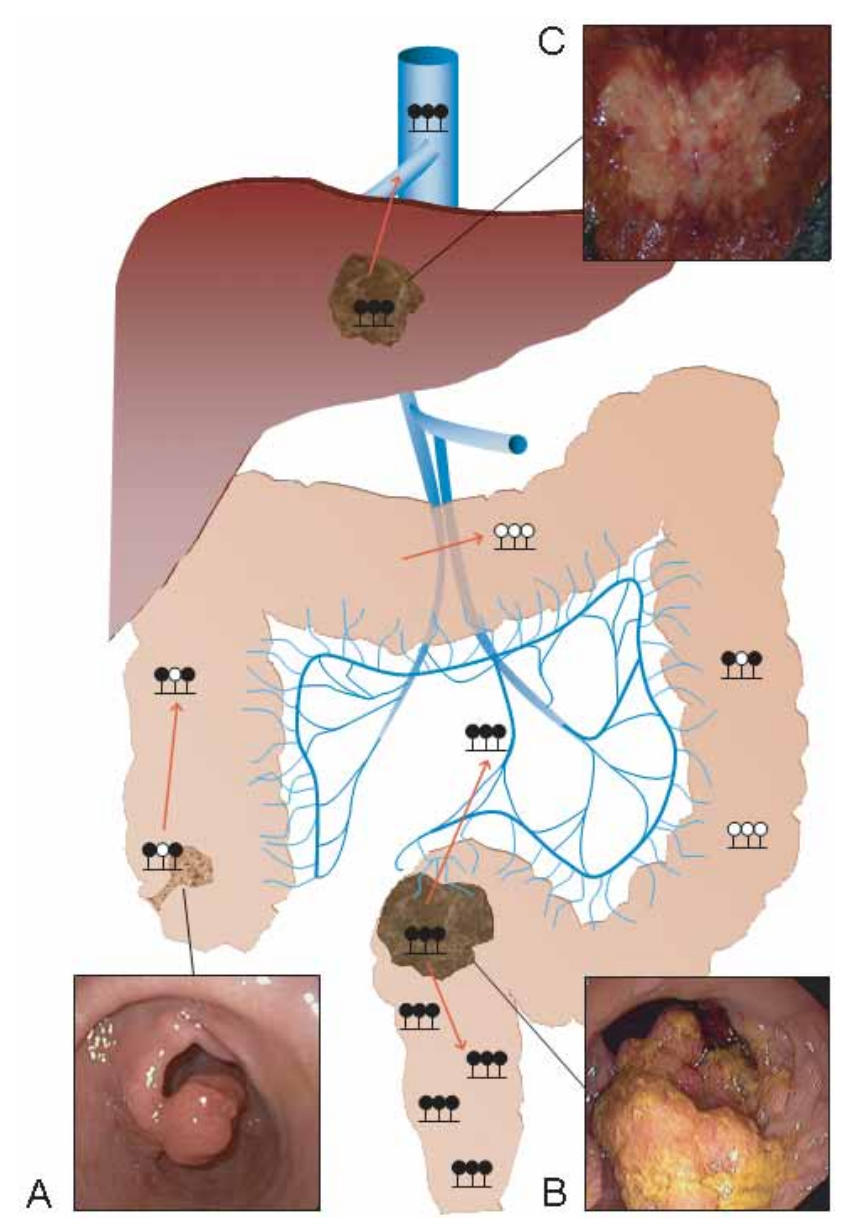

Fig. 1. Methylated DNA can be detected in tumor-derived DNA found either in the bloodstream of CRC patients or in samples drained to the outside of the body (e.g. stool). Three different situations are demonstrated: (A) colorectal polyp; (B) CRC and (C) metastasized CRC (e.g. after primary curative resection). Fully methylated (tumor-specific) DNA is represented by black lollipops, hemi-methylated (tumor-specific) DNA is represented by black mixed with white lollipops and normal DNA is represented be solely white ones. In situations (A) and (B) it seems that methylated DNA can be more sufficiently detected in stool samples, because tumor-specific methylated DNA is shed into the gut lumen and is then be transported to the outside of the body by the physiological bowel movement. It seems that less tumor-derived DNA will be transported into the bloodstream, especially in terms of pre-neoplastic lesions (which are not invasive by definition). Situation (C) represents metastasized CRC (e.g. after primary curative resection). The life-threatening event in CRC is not lymph node metastasis per se, but hematogenous metastases which mainly affect the liver or the lung. Therefore, it seems plausible to use methylated tumor-derived DNA, especially in patients bloodstream for early detection of recurrent diesease. DNA methylation changes in CRC patients may serve, on the one hand, as a possible new screening marker for CRC (fecal DNA; maybe in combination with bloodstream testing) and, on the other hand, as a tool for therapy monitoring and early detection of recurrent disease (bloodstream testing) in patients having had CRC.

nation of both (tubulovillous adenomas). The time required for the development of malignancy from adenoma is lengthy, with even conservative estimates indicating an interval of 5-10 years [60]. Moreover, only a small percentage of adenomas progress to carcinoma. Although there is presently no clear way to identify which adenomas will become malignant, subsequent progression is associated with severe dysplasia, patient age, size of adenoma and histological type [61]. Adenomas that are $>1 \mathrm{~cm}$, show severe dysplasia and/or a villous architecture are described as advanced [23].
The average time for an asymptomatic early CRC to become an advanced symptomatic lesion is thought to be around 2-3 years [62]. Additionally, survival from CRC is intimately related to its stage, with early CRC having an excellent outcome [62].

De novo CRCs [63] are typically superficial and flat (non-polyploid), with no detectable adenomatous remnants. Interestingly, these tumors might evolve through a distinct genetic pathway, in which the frequency of KRAS mutations is lower than in the adenomacarcinoma sequence [64-66]. Approximately 15\% 
Table 2a

DNA methylation in CRC cell lines

\begin{tabular}{|c|c|c|c|c|c|c|c|}
\hline Gene & Name & CRC cell line & $\begin{array}{l}\begin{array}{l}\text { Promotor } \\
\text { methylation } \\
\text { status }\end{array} \\
\end{array}$ & $\begin{array}{l}\text { Gene } \\
\text { expression }\end{array}$ & $\begin{array}{l}\text { Gene expression } \\
\text { after treatment }\end{array}$ & $\begin{array}{l}\text { Technique } \\
\text { used }\end{array}$ & Reference \\
\hline APC & $\begin{array}{l}\text { adenomatosis polyposis } \\
\text { coli }\end{array}$ & $\begin{array}{l}\text { DLD-1, SW480, Colo320, } \\
\text { HT29, Colo201 }\end{array}$ & HM & No & & MSP & $\begin{array}{l}\text { Sakamoto } \text { et al. (2001) } \\
{[116]}\end{array}$ \\
\hline APC & $\begin{array}{l}\text { adenomatosis polyposis } \\
\text { coli }\end{array}$ & $\begin{array}{l}11 \text { MSS CRC-CL } \\
9 \text { MSI CRC-CL }\end{array}$ & $\begin{array}{l}\text { HM } 2 / 11 \\
\text { HM } 2 / 9\end{array}$ & & & MSP & Lind et al. (2004) [105] \\
\hline BNIP3 & $\begin{array}{l}\text { BCL2/adenovirus E1B } \\
\text { 19kDa interacting protein } 3\end{array}$ & $\begin{array}{l}\text { RKO, DLD-1, SW48, } \\
\text { SW480, HT29, CoLo320 }\end{array}$ & HM & No & Yes/5AdC & COBRA & Murai et al. (2005) [117] \\
\hline CDH1 & $\begin{array}{l}\text { Cadherin 1, type 1, E- } \\
\text { cadherin (epithelial) }\end{array}$ & $\begin{array}{l}11 \text { MSS CRC-CL } \\
9 \text { MSI CRC-CL }\end{array}$ & $\begin{array}{l}/ 11 \\
2 / 9\end{array}$ & & & MSP & Lind et al. (2004) [105] \\
\hline $\mathrm{CDH} 13$ & Cadherin $13, \mathrm{H}$-cadherin & $13 \mathrm{CRC}-\mathrm{CL}$ & $\begin{array}{l}\text { HM 7/13 } \\
(54 \%)\end{array}$ & No in $6 / 7$ & $4 / 4 \mathrm{Yes} / 5 \mathrm{AdC}$ & MSP & $\begin{array}{l}\text { Toyooka et al. (2002) } \\
\text { [118] }\end{array}$ \\
\hline $\mathrm{CDH} 4$ & Cadherin 4, R-cadherin & RKO & $\mathrm{HM}$ & no & Yes/ $5 \mathrm{AdC}$ & MSP & Miotto et al. (2004) [119] \\
\hline $\begin{array}{l}\text { CDNK2A } \\
\left(\mathrm{P} 14^{\mathrm{ARF}}\right)\end{array}$ & $\begin{array}{l}\text { cyclin-dependent kinase } \\
\text { inhibitor } 2 \mathrm{~A}\end{array}$ & DLD-1, HCT-15, SW48 & HM & No & Yes/5dAC & MSP & Zheng et al. (2000) [120] \\
\hline $\begin{array}{l}\text { CDNK2A } \\
\left(\mathrm{P} 14^{\mathrm{ARF}}\right)\end{array}$ & $\begin{array}{l}\text { cyclin-dependent kinase } \\
\text { inhibitor } 2 \mathrm{~A}\end{array}$ & $\begin{array}{l}11 \text { MSS CRC-CL } \\
9 \text { MSI CRC-CL }\end{array}$ & $\begin{array}{l}5 / 11 \\
8 / 9\end{array}$ & & & MSP & Lind et al. (2004) [105] \\
\hline $\begin{array}{l}\text { CDNK2A } \\
\text { (P16) }\end{array}$ & $\begin{array}{l}\text { cyclin-dependent kinase } \\
\text { inhibitor } 2 \mathrm{~A}\end{array}$ & Six CRC-CL & $5 / 6$ & & & MSP & $\begin{array}{l}\text { Wagner } \text { et al. (2002) } \\
\text { [121] }\end{array}$ \\
\hline $\begin{array}{l}\text { CDNK2A } \\
\text { (p16INK4A) }\end{array}$ & $\begin{array}{l}\text { cyclin-dependent kinase } \\
\text { inhibitor } 2 \mathrm{~A}\end{array}$ & $\begin{array}{l}11 \text { MSS CRC-CL } \\
9 \text { MSI CRC-CL }\end{array}$ & $\begin{array}{l}8 / 11 \\
7 / 9\end{array}$ & & & MSP & Lind et al. (2004) [105] \\
\hline CDX1 & $\begin{array}{l}\text { caudal type homeo box } \\
\text { transcription factor } 1\end{array}$ & $37 \mathrm{CRC}-\mathrm{CL}$ & $\begin{array}{l}\text { HM 7/37 } \\
(19 \%)\end{array}$ & No & $5 / 5 \mathrm{Yes} / 5 \mathrm{AdC}$ & MSP & $\begin{array}{l}\text { Wong et al. (2004) } \\
{[122]}\end{array}$ \\
\hline CDX1 & $\begin{array}{l}\text { caudal type homeo box } \\
\text { transcription factor } 1\end{array}$ & $\begin{array}{l}\text { CaCo2, SW480, HCT116, } \\
\text { ColoDM, DLD-1, RKO }\end{array}$ & HM & No & Yes/5AdC & MSP & Suh et al. (2002) [123] \\
\hline CHFR & $\begin{array}{l}\text { heckpoint with forkhead } \\
\text { and ring finger domains }\end{array}$ & HCT116, DLD1, HT29 & HM & No/reduced & Yes $/ 5 \mathrm{AdC}$ & MSP & Corn et al. (2003) [124] \\
\hline COL1A2 & Collagen, typeI, alpha2(I) & HCT116, SW480, SW620 & HM & No & Yes/5AdC & & $\begin{array}{l}\text { Sengupta } \text { et al. (2003) } \\
{[125]}\end{array}$ \\
\hline DAPK1 & $\begin{array}{l}\text { Death associated protein } \\
\text { kinasel }\end{array}$ & Nine CRC-CL & $3 / 9$ & No in $5 / 9$ & & COBRA & Satoh et al. (2002) [126] \\
\hline EphA7 & EPH receptor A7 & DLD-1, HCT116, SW620 & HM & No & Yes/5AdC & MSP & Wang et al. (2005) [127] \\
\hline GATA-4 & GATA binding protein 4 & $\begin{array}{l}\text { RKO, DLD1, HCT116, } \\
\text { HT29, LoVo, SW480 }\end{array}$ & HM & No in $4 / 6$ & Yes/5AdC & MSP & $\begin{array}{l}\text { Akiyama et al. (2003) } \\
{[128]}\end{array}$ \\
\hline GATA-5 & GATA binding protein 5 & $\begin{array}{l}\text { RKO, DLD1, HCT116, } \\
\text { HT29, LoVo, SW480 }\end{array}$ & HM & No in $5 / 6$ & Yes/5AdC & MSP & $\begin{array}{l}\text { Akiyama et al. (2003) } \\
{[128]}\end{array}$ \\
\hline ID4 & inhibitor of DNA binding 4 & SW480, DLD1 & HM & No & Yes/5Aza & MSP & $\begin{array}{l}\text { Umetani } \text { et al. (2004) } \\
\text { [129] }\end{array}$ \\
\hline MGMT & $\begin{array}{l}\text { O-6-methylguanine-DNA } \\
\text { methyltransferase }\end{array}$ & $\begin{array}{l}11 \text { MSS CRC-CL } \\
9 \text { MSI CRC-CL }\end{array}$ & $\begin{array}{l}5 / 11 \\
5 / 9\end{array}$ & & & MSP & Lind et al. (2004) [105] \\
\hline MLH1 & $\begin{array}{l}\text { MutL homolog 1, colon } \\
\text { cancer, nonpolyposis type } \\
2\end{array}$ & $\begin{array}{l}11 \text { MSS CRC-CL } \\
9 \text { MSI CRC-CL }\end{array}$ & $\begin{array}{l}0 / 11 \\
3 / 9\end{array}$ & & & MSP & Lind et al. (2004) [105] \\
\hline PTGIS & $\begin{array}{l}\text { Prostaglandin I2 } \\
\text { (prostacyclin) synthase }\end{array}$ & HCT116 & HM & No & Yes $/ 5 \mathrm{dAC}+\mathrm{HDAC}$ & $\mathrm{BS}$ & \begin{tabular}{|l|l} 
Frigola et al. (2005) [130] \\
\end{tabular} \\
\hline $\begin{array}{l}\text { PTGS2 } \\
(\text { Cox-2) }\end{array}$ & $\begin{array}{l}\text { prostaglandin- } \\
\text { endoperoxide synthase } 2 \\
\text { (Cyclooxygenase) }\end{array}$ & Eight CRC-CL & $\begin{array}{l}\text { HM } 1 / 8 \\
\text { (RKO) }\end{array}$ & No & Yes $/ 5 \mathrm{AdC}$ & COBRA & Toyota et al. (2000) [131] \\
\hline RASSF1 & $\begin{array}{l}\text { Ras association } \\
\text { (RalGDS/AF-6) domain } \\
\text { family } 1\end{array}$ & Five CRC-CL & HM $4 / 5$ & & & MSP & $\begin{array}{l}\text { Wagner } \text { et al. (2002) } \\
{[121]}\end{array}$ \\
\hline RASSF2 & $\begin{array}{l}\text { Ras association } \\
\text { (RalGDS/AF-6) domain } \\
\text { family } 2\end{array}$ & LOVO, DLD-1, RKO & HM & No & Yes $/ 5$ AdC & COBRA & $\begin{array}{l}\text { Akino et al. (2005) } \\
{[132]}\end{array}$ \\
\hline RUNX3 & $\begin{array}{l}\text { runt-related transcription } \\
\text { factor } 3\end{array}$ & $32 \mathrm{CRC}-\mathrm{CL}$ & HM $12 / 32$ & No & Yes/5AdC & MSP & Ku et al. (2004) [133] \\
\hline SFRP1 & $\begin{array}{l}\text { Secreted frizzled-related } \\
\text { protein } 1\end{array}$ & $\begin{array}{l}\text { RKO, DLD-1, HCT116, } \\
\text { LOVO, SW480 }\end{array}$ & HM & No & Yes/5AdC & MSP & Suzuki et al. (2002) [108] \\
\hline SLC5A8 & $\begin{array}{l}\text { solute carrier family } 5 \\
\text { (iodide transporter), } \\
\text { member } 8\end{array}$ & $31 \mathrm{CRC}-\mathrm{CL}$ & HM 16/31 & $\begin{array}{l}\text { No in } \\
23 / 31\end{array}$ & $6 / 8 \mathrm{Yes} / 5 \mathrm{Aza}$ & MSP & Li et al. (2003) [101] \\
\hline $\begin{array}{l}\text { SMARCA3 } \\
\text { (HLTF) }\end{array}$ & $\begin{array}{l}\text { SWI/SNF related, matrix } \\
\text { associated, actin dependent } \\
\text { regulator of chromatin, } \\
\text { subfamily a, member } 3\end{array}$ & 34 CRC-CL & HM 9/34 & No in $9 / 9$ & Yes/5Aza & MSP & $\begin{array}{l}\text { Moinova et al. (2002) } \\
\text { [134] }\end{array}$ \\
\hline \begin{tabular}{l|} 
TMEFF2 \\
(TPEF/HPP1)
\end{tabular} & $\begin{array}{l}\text { transmembrane protein } \\
\text { with EGF-like and two } \\
\text { follistatin-like domains } 2\end{array}$ & LOVO, DLD-1 & HM & No & Yes/5AdC & $\mathrm{ML}$ & Ebert et al. (2005) [135] \\
\hline $\begin{array}{l}\text { TMEFF2 } \\
\text { (TPEF/HPP1) }\end{array}$ & $\begin{array}{l}\text { transmembrane protein } \\
\text { with EGF-like and two } \\
\text { follistatin-like domains } 2\end{array}$ & HT29, LOVO & HM & No & Yes/5AdC & COBRA & Young et al. (2001) [136] \\
\hline
\end{tabular}

of CRCs are familial, with autosomal-dominant and autosomal-recessive modes of inheritance. The most common inherited conditions are familial adenomatous polyposis (FAP) and hereditary non-polyposis colorectal cancer (HNPCC). It arises because of mutations in mismatch-repair genes, including $\mathrm{MLH1}, \mathrm{MSH} 2$, MSH3, MSH6, PMS1 and PMS2 [67]. The loss of repar- ative mechanisms leads to an increased mutation rate especially in repetitive DNA sequences (microsatellite sequences), resulting in microsatellite instability (MSI) [68,69]. For example, BAT26 (big adenine tract 26) is a microsatellite region that is altered in almost all mismatch-repair-deficient CRCs.

Additional mutated genes that can be detected in 
Table $2 b$

DNA methylation in CRC tissue

\begin{tabular}{|c|c|c|c|c|c|c|c|}
\hline Gene & Name & \begin{tabular}{|l|} 
Tissue specimen \\
\end{tabular} & Histological type & $\begin{array}{l}\begin{array}{l}\text { Fraction } \\
\text { methylated }\end{array} \\
\end{array}$ & $\begin{array}{l}\text { Percentage } \\
\text { methylated }\end{array}$ & $\begin{array}{l}\text { Technique } \\
\text { used }\end{array}$ & Reference \\
\hline $\mathrm{ABCB} 1$ & $\begin{array}{l}\text { ATP-binding cassette, sub-family } \\
\text { B (MDR/TAP), member } 1\end{array}$ & \begin{tabular}{|l|} 
Formalin-fixed, \\
paraffin-embedded \\
or fresh frozen
\end{tabular} & CRC & $67 / 275$ & 24 & MSP & $\begin{array}{l}\text { Van Rijnsoever } \text { et al. } \\
\text { (2002) [137] }\end{array}$ \\
\hline $\begin{array}{l}\text { APBA1 } \\
\text { (MINT1) }\end{array}$ & $\begin{array}{l}\text { amyloid beta (A4) precursor } \\
\text { protein-binding, family A, } \\
\text { member 1 } \\
\text { (Methylated in Tumor 1) } \\
\end{array}$ & $\begin{array}{l}\text { Endoscopic biopsy } \\
\text { specimen }\end{array}$ & $\begin{array}{l}\text { Serrated Adenomas } \\
\text { Tubular Adenomas } \\
\text { Normal Tissue }\end{array}$ & $\begin{array}{l}11 / 27 \\
3 / 34 \\
0 / 16\end{array}$ & $\begin{array}{l}40 \\
9 \\
0\end{array}$ & MSP & Park et al. (2003) [138] \\
\hline $\begin{array}{l}\text { APBA2 } \\
\text { (MINT2) }\end{array}$ & $\begin{array}{l}\text { amyloid beta (A4) precursor } \\
\text { protein-binding, family A, } \\
\text { member } 2 \\
\text { (Methylated in Tumor 2) }\end{array}$ & $\begin{array}{l}\text { Endoscopic biopsy } \\
\text { specimen }\end{array}$ & $\begin{array}{l}\text { Serrated Adenomas } \\
\text { Tubular Adenomas } \\
\text { Normal Tissue }\end{array}$ & $\begin{array}{l}17 / 27 \\
6 / 34 \\
1 / 17\end{array}$ & $\begin{array}{l}63 \\
18 \\
6\end{array}$ & MSP & Park et al. (2003) [138] \\
\hline $\begin{array}{l}\text { APBA2 } \\
\text { (MINT2) }\end{array}$ & $\begin{array}{l}\text { amyloid beta (A4) precursor } \\
\text { protein-binding, family A, } \\
\text { member } 2 \\
\text { (Methylated in Tumor 2) }\end{array}$ & $\begin{array}{l}\text { Formalin-fixed, } \\
\text { paraffin-embedded } \\
\text { or fresh frozen }\end{array}$ & CRC & $102 / 275$ & 37 & MSP & $\begin{array}{l}\text { Van Rijnsoever } \text { et al. } \\
(2002)[137]\end{array}$ \\
\hline $\mathrm{APC}$ & adenomatosis polyposis coli & & $\begin{array}{l}25 \text { MSS CRC } \\
28 \text { MSI CRC } \\
\end{array}$ & $\begin{array}{l}7 / 25 \\
10 / 28 \\
\end{array}$ & $\begin{array}{l}28 \\
36 \\
\end{array}$ & MSP & Lind et al. (2004) [105] \\
\hline $\mathrm{APC}$ & adenomatosis polyposis coli & & $\begin{array}{l}\text { Normal tissue } \\
\text { CRC } \\
\text { Liver metastasis }\end{array}$ & $\begin{array}{l}0 / 21 \\
10 / 47 \\
10 / 24\end{array}$ & \begin{tabular}{|l|}
0 \\
21 \\
42
\end{tabular} & $\overline{\mathrm{ML}}$ & Ebert et al. (2005) [135] \\
\hline $\mathrm{APC}$ & adenomatosis polyposis coli & Fresh frozen & $\begin{array}{l}\text { CRC } \\
\text { Adenoma } \\
\end{array}$ & $\begin{array}{l}3 / 31 \\
3 / 40\end{array}$ & $\begin{array}{l}9 \\
8 \\
\end{array}$ & MSP & Kim et al. (2003) [139] \\
\hline $\mathrm{APC}$ & adenomatosis polyposis coli & $\begin{array}{l}\text { Formalin-fixed, } \\
\text { paraffin-embedded }\end{array}$ & $\begin{array}{l}\text { CRC } \\
\text { Normal Tissue }\end{array}$ & & $\begin{array}{l}7,4 \\
3,3 \\
\end{array}$ & MSP & Xiong et al. (2001) [140] \\
\hline APC & adenomatosis polyposis coli & Fresh frozen & $\begin{array}{l}\text { CRC } \\
\text { Normal tissue } \\
\text { Adenomas } \\
\end{array}$ & $\begin{array}{l}20 / 108 \\
0 / 28 \\
9 / 48 \\
\end{array}$ & \begin{tabular}{|l|l|}
18 \\
0 \\
18 \\
\end{tabular} & MSP & $\begin{array}{l}\text { Esteller } \text { et al. (2000) } \\
\text { [141] }\end{array}$ \\
\hline AXIN-2 & AXIN 2 (CONDUCTIN, AXIL) & Fresh tissue & $\begin{array}{l}\text { MSI CRC } \\
\text { MSS CRC }\end{array}$ & $\begin{array}{l}5 / 10 \\
0 / 10\end{array}$ & \begin{tabular}{l|}
50 \\
0
\end{tabular} & HDO-MA & $\begin{array}{l}\text { Koinuma et al. (2006) } \\
\text { [106] }\end{array}$ \\
\hline BNIP3 & $\begin{array}{l}\text { BCL2/adenovirus E1B 19kDa } \\
\text { interacting protein } 3\end{array}$ & & CRC & $40 / 61$ & 66 & COBRA & Murai et al. (2005) [117] \\
\hline CDH1 & $\begin{array}{l}\text { Cadherin 1, type 1, E-cadherin } \\
\text { (epithelial) }\end{array}$ & & $\begin{array}{l}24 \text { MSS CRC } \\
28 \text { MSI CRC }\end{array}$ & $\begin{array}{l}10 / 24 \\
11 / 28 \\
\end{array}$ & $\begin{array}{l}42 \\
39 \\
\end{array}$ & MSP & Lind et al. (2004) [105] \\
\hline $\mathrm{CDHl}$ & $\begin{array}{l}\text { cadherin 1, type 1, E-cadherin } \\
\text { (epithelial) }\end{array}$ & $\begin{array}{l}\text { Formalin-fixed, } \\
\text { paraffin-embedded } \\
\text { or fresh frozen }\end{array}$ & CRC & $12 / 22$ & 54,5 & MSP & $\begin{array}{l}\text { Kanazawa et al. (2002) } \\
{[142]}\end{array}$ \\
\hline $\mathrm{CDH1}$ & $\begin{array}{l}\text { Cadherin 1, type 1, E-cadherin } \\
\text { (epithelial) }\end{array}$ & Fresh frozen & CRC & $34 / 61$ & 56 & MSP & Garinis et al. (2002) [143] \\
\hline $\mathrm{CDH13}$ & $\mathrm{H}$-Cadherin & Fresh frozen & CRC & $23 / 61$ & 38 & MSP & Hibi et al. (2005) [144] \\
\hline CDH13 & H-cadherin & Fresh frozen & CRC & $27 / 84$ & 32 & MSP & Hibi et al. (2004) [145] \\
\hline CDH13 & H-cadherin & Fresh tissue & $\begin{array}{l}\text { CRC } \\
\text { Adenoma } \\
\text { Non-malignant mucosa } \\
\text { CRC-CL }\end{array}$ & $\begin{array}{l}17 / 35 \\
8 / 19 \\
2 / 33 \\
7 / 13 \\
\end{array}$ & $\begin{array}{l}49 \\
42 \\
6 \\
54 \\
\end{array}$ & MSP & $\begin{array}{l}\text { Toyooka et al. (2002) } \\
\text { [118] }\end{array}$ \\
\hline $\mathrm{CDH} 4$ & Cadherin 4, R-cadherin & & $\begin{array}{l}\text { CRC } \\
\text { Normal tissue f. CRC-Pat } \\
\text { Adenoma } \\
\text { Normal tissue }\end{array}$ & $\begin{array}{l}38 / 49 \\
5 / 17 \\
10 / 10 \\
0 / 10\end{array}$ & $\begin{array}{l}78 \\
29 \\
100 \\
0\end{array}$ & MSP & Miotto et al. (2004) [119] \\
\hline CDKN2A & $\begin{array}{l}\text { cyclin-dependent kinase inhibitor } \\
2 \mathrm{~A}\end{array}$ & $\begin{array}{l}\text { Formalin-fixed, } \\
\text { paraffin-embedded }\end{array}$ & Adenomas & $14 / 41$ & 34 & MSP & Petko et al. (2005) [96] \\
\hline $\begin{array}{l}\text { CDNK2A } \\
\text { (P16) }\end{array}$ & $\begin{array}{l}\text { cyclin-dependent kinase inhibitor } \\
2 \mathrm{~A}\end{array}$ & Fresh frozen & $\begin{array}{l}\text { CRC Duke's A, B } \\
\text { CRC Duke's C,C } \\
\text { Total }\end{array}$ & $\begin{array}{l}4 / 33 \\
22 / 29 \\
26 / 62 \\
\end{array}$ & $\begin{array}{l}12 \\
76 \\
42 \\
\end{array}$ & MSP & Yi et al. (2001) [146] \\
\hline $\begin{array}{l}\text { CDNK2A } \\
\text { (P16) }\end{array}$ & $\begin{array}{l}\text { cyclin-dependent kinase inhibitor } \\
2 \mathrm{~A}\end{array}$ & $\begin{array}{l}\text { Formalin-fixed, } \\
\text { paraffin-embedded } \\
\text { or fresh frozen }\end{array}$ & CRC & $100 / 275$ & 36 & MSP & $\begin{array}{l}\text { Van Rijnsoever } \text { et al. } \\
\text { (2002) [137] }\end{array}$ \\
\hline $\begin{array}{l}\text { CDNK2A } \\
\text { (P16) }\end{array}$ & $\begin{array}{l}\text { cyclin-dependent kinase inhibitor } \\
2 \mathrm{~A}\end{array}$ & $\begin{array}{l}\text { Formalin-fixed, } \\
\text { paraffin-embedded }\end{array}$ & $\begin{array}{l}\text { CRC } \\
\text { Normal Tissue }\end{array}$ & $\begin{array}{l}62 \\
62\end{array}$ & $\begin{array}{l}3,8 \\
2,3\end{array}$ & MSP & Xiong et al. (2001) [140] \\
\hline $\begin{array}{l}\text { CDNK2A } \\
\text { (P16) }\end{array}$ & $\begin{array}{l}\text { cyclin-dependent kinase inhibitor } \\
2 \mathrm{~A}\end{array}$ & & CRC & $6 / 29$ & 21 & MSP & $\begin{array}{l}\text { Wagner } \text { et al. (2002) } \\
\text { [121] }\end{array}$ \\
\hline $\begin{array}{l}\text { CDNK2A } \\
\text { (P16) }\end{array}$ & $\begin{array}{l}\text { cyclin-dependent kinase inhibitor } \\
2 \mathrm{~A}\end{array}$ & $\begin{array}{l}\text { Endoscopic biopsy } \\
\text { specimen }\end{array}$ & $\begin{array}{l}\text { Serrated Adenomas } \\
\text { Tubular Adenomas } \\
\text { Normal Tissue }\end{array}$ & $\begin{array}{l}11 / 27 \\
10 / 34 \\
0 / 16 \\
\end{array}$ & $\begin{array}{l}40 \\
29 \\
0 \\
\end{array}$ & MSP & Park et al. (2003) [138] \\
\hline $\begin{array}{l}\text { CDNK2A } \\
\text { (P16) }\end{array}$ & $\begin{array}{l}\text { cyclin-dependent kinase inhibitor } \\
2 \mathrm{~A}\end{array}$ & & $\begin{array}{l}\text { CRC (88) } \\
\text { CIMP+ } \\
\text { CIMP- } \\
\text { Adenoma (45) } \\
\text { CIMP+ } \\
\text { CIMP- }\end{array}$ & $\begin{array}{l}21 / 41 \\
0 / 47 \\
12 / 22 \\
0 / 23\end{array}$ & $\begin{array}{l}51 \\
0 \\
55 \\
0\end{array}$ & $\begin{array}{l}\text { MSP } \\
\text { BS }\end{array}$ & Toyota et al. (2000) [147] \\
\hline $\begin{array}{l}\text { CDNK2A } \\
\text { (P16) }\end{array}$ & $\begin{array}{l}\text { cyclin-dependent kinase inhibitor } \\
2 \mathrm{~A}\end{array}$ & Fresh frozen & Liver metastasis & $8 / 11$ & 73 & MSP & $\begin{array}{l}\text { Nakayama et al. (2003) } \\
{[148]}\end{array}$ \\
\hline $\begin{array}{l}\text { CDNK2A } \\
\text { (P16) }\end{array}$ & $\begin{array}{l}\text { cyclin-dependent kinase inhibitor } \\
2 \mathrm{~A}\end{array}$ & Fresh frozen & CRC & $20 / 52$ & 38 & MSP & Zou et al. (2002) [114] \\
\hline $\begin{array}{l}\text { CDNK2A } \\
\text { (P16) }\end{array}$ & $\begin{array}{l}\text { cyclin-dependent kinase inhibitor } \\
\text { 2A }\end{array}$ & Fresh frozen & CRC & $44 / 94$ & 47 & MSP & $\begin{array}{l}\text { Nakayama et al. (2002) } \\
\text { [113] }\end{array}$ \\
\hline $\begin{array}{l}\text { CDNK2A } \\
\text { (P16) }\end{array}$ & $\begin{array}{l}\text { cyclin-dependent kinase inhibitor } \\
2 \mathrm{~A}\end{array}$ & Fresh frozen & CRC & $31 / 58$ & 53 & MSP & $\begin{array}{l}\text { Lecomte et al. (2002) } \\
\text { [115] }\end{array}$ \\
\hline $\begin{array}{l}\text { CDNK2A } \\
\left(\mathrm{P} 14^{\mathrm{ARF}}\right)\end{array}$ & $\begin{array}{l}\text { cyclin-dependent kinase inhibitor } \\
2 \mathrm{~A}\end{array}$ & Fresh frozen & $\begin{array}{l}\text { Adenoma from: } \\
\text { FAP-patient } \\
\text { Multiple Adenoma patient } \\
\text { MSI-H CRC-Pat. } \\
\text { MSS/MSI-L CRC-Pat. }\end{array}$ & $\begin{array}{l}13 / 32 \\
20 / 29 \\
12 / 14 \\
14 / 16\end{array}$ & $\begin{array}{l}41 \\
69 \\
86 \\
88\end{array}$ & MSP & $\begin{array}{l}\text { Wynter } \text { et al. (2006) } \\
{[149]}\end{array}$ \\
\hline $\begin{array}{l}\text { CDNK2A } \\
\left(\mathrm{P} 14^{\mathrm{ARF}}\right)\end{array}$ & $\begin{array}{l}\text { cyclin-dependent kinase inhibitor } \\
2 \mathrm{~A}\end{array}$ & & $\begin{array}{l}24 \text { MSS CRC } \\
28 \text { MSI CRC } \\
\end{array}$ & $\begin{array}{l}3 / 24 \\
17 / 28 \\
\end{array}$ & $\begin{array}{l}12 \\
39 \\
\end{array}$ & MSP & Lind et al. (2004) [105] \\
\hline $\begin{array}{l}\text { CDNK2A } \\
\left(\mathrm{P} 14^{\mathrm{ARF}}\right)\end{array}$ & $\begin{array}{l}\text { cyclin-dependent kinase inhibitor } \\
2 \mathrm{~A}\end{array}$ & & $\begin{array}{l}\text { CRC } \\
\text { Dysplasia } \\
\text { UC mucosa } \\
\text { Normal Tissue }\end{array}$ & $\begin{array}{l}19 / 38 \\
4 / 12 \\
3 / 5 \\
3 / 40\end{array}$ & $\begin{array}{l}50 \\
33 \\
60 \\
3,7 \\
\end{array}$ & $\begin{array}{l}\text { MSP } \\
\text { BS }\end{array}$ & Sato et al. (2002) [86] \\
\hline
\end{tabular}


Table $2 b$, continued

\begin{tabular}{|c|c|c|c|c|c|c|c|}
\hline $\begin{array}{l}\text { CDNK2A } \\
\text { (p16INK4A) }\end{array}$ & $\begin{array}{l}\text { cyclin-dependent kinase inhibitor } \\
\text { 2A }\end{array}$ & Fresh frozen & $\begin{array}{l}\text { Adenoma from: } \\
\text { FAP-patient } \\
\text { Multiple Adenoma patient } \\
\text { MSI-H CRC-Pat. } \\
\text { MSS/MSI-L CRC-Pat. }\end{array}$ & $\begin{array}{l}17 / 33 \\
13 / 29 \\
9 / 14 \\
10 / 16\end{array}$ & $\begin{array}{l}52 \\
45 \\
64 \\
63 \\
\end{array}$ & MSP & $\begin{array}{l}\text { Wynter et al. (2006) } \\
\text { [149] }\end{array}$ \\
\hline $\begin{array}{l}\text { CDNK2A } \\
\text { (p16INK4A) }\end{array}$ & $\begin{array}{l}\text { cyclin-dependent kinase inhibitor } \\
\text { 2A }\end{array}$ & & $\begin{array}{l}25 \text { MSS CRC } \\
28 \text { MSI CRC }\end{array}$ & $\begin{array}{l}7 / 25 \\
10 / 28 \\
\end{array}$ & $\begin{array}{l}28 \\
36\end{array}$ & MSP & Lind et al. (2004) [105] \\
\hline CHFR & $\begin{array}{l}\text { checkpoint with forkhead and ring } \\
\text { finger domains }\end{array}$ & $\begin{array}{l}\text { Fresh frozen and } \\
\text { paraffin-embedded }\end{array}$ & $\begin{array}{l}\text { CRC } \\
\text { Normal tissue }\end{array}$ & $\begin{array}{l}11 / 30 \\
2 / 9\end{array}$ & $\begin{array}{l}37 \\
22 \\
\end{array}$ & MSP & Corn et al. (2003) [124] \\
\hline COL1A2 & Collagen, typeI, alpha2(I) & Fresh frozen & CRC & $5 / 6$ & 83 & MSP & $\begin{array}{l}\text { Sengupta et al. (2003) } \\
\text { [125] }\end{array}$ \\
\hline DAPK1 & Death associated protein kinase 1 & CRC & & $4 / 28$ & 14 & COBRA & Satoh et al. (2002) [126] \\
\hline DAPK1 & \begin{tabular}{|l|} 
Death associated protein kinase 1 \\
\end{tabular} & Fresh frozen & $\begin{array}{l}\text { CRC patients } \\
\text { Normal tissue }\end{array}$ & $\begin{array}{l}67 / 122 \\
0 / 10\end{array}$ & \begin{tabular}{|l|}
55 \\
0
\end{tabular} & MSP & $\begin{array}{l}\text { Yamaguchi et al. (2003) } \\
{[150]}\end{array}$ \\
\hline EphA7 & EPH receptor A7 & $\begin{array}{l}\text { Formalin-fixed, } \\
\text { paraffin-embedded }\end{array}$ & CRC & $37 / 75$ & 49 & MSP & Wang et al. (2005) [127] \\
\hline ESR1 & Estrogen Receptor 1 & Fresh frozen & $\begin{array}{l}\text { UC with neoplasia } \\
\text { UC without neoplasia }\end{array}$ & & \begin{tabular}{|l|}
$25.4-17.8$ \\
$4.0-6.4$ \\
\end{tabular} & COBRA & $\begin{array}{l}\text { Tominaga et al. (2005) } \\
\text { [151] }\end{array}$ \\
\hline ESR1 & Estrogen Receptor 1 & $\begin{array}{l}\text { Formalin-fixed, } \\
\text { paraffin-embedded }\end{array}$ & $\begin{array}{l}\text { CRC } \\
\text { Normal Tissue }\end{array}$ & & $\begin{array}{l}28,1 \\
7,0\end{array}$ & MSP & Xiong et al. (2001) [140] \\
\hline GATA-4 & GATA binding protein 4 & & CRC & $30 / 45$ & 67 & MSP & $\begin{array}{l}\text { Akiyama et al. (2003) } \\
{[128]}\end{array}$ \\
\hline GATA-5 & \begin{tabular}{|l|} 
GATA binding protein 5 \\
\end{tabular} & & CRC & $28 / 44$ & 64 & MSP & $\begin{array}{l}\text { Akiyama et al. (2003) } \\
{[128]}\end{array}$ \\
\hline ID4 & inhibitor of DNA binding 4 & $\begin{array}{l}\text { Formalin-fixed, } \\
\text { paraffin-embedded }\end{array}$ & $\begin{array}{l}\text { Normal tissue } \\
\text { Adenoma } \\
\text { CRC } \\
\text { Liver-metastasis }\end{array}$ & $\begin{array}{l}0 / 9 \\
0 / 13 \\
49 / 92 \\
19 / 26\end{array}$ & \begin{tabular}{|l|}
0 \\
0 \\
53 \\
73
\end{tabular} & MSP & $\begin{array}{l}\text { Umetani et al. (2004) } \\
\text { [129] }\end{array}$ \\
\hline MGMT & $\begin{array}{l}\text { O-6-methylguanine-DNA } \\
\text { methyltransferase }\end{array}$ & $\begin{array}{l}\text { Formalin-fixed, } \\
\text { paraffin-embedded }\end{array}$ & $\begin{array}{ll}\text { Adenomas } & 19 / 39\end{array}$ & & 49 & MSP & Petko et al. (2005) [96] \\
\hline MGMT & $\begin{array}{l}\text { O-6-methylguanine-DANN } \\
\text { methyltransferase }\end{array}$ & & $\begin{array}{l}25 \text { MSS CRC } \\
28 \text { MSI CRC }\end{array}$ & $\begin{array}{l}10 / 25 \\
11 / 28 \\
\end{array}$ & $\begin{array}{l}40 \\
39 \\
\end{array}$ & MSP & Lind et al. (2004) [105] \\
\hline MGMT & $\begin{array}{l}\text { O-6-methylguanine-DANN } \\
\text { methyltransferase }\end{array}$ & Fresh frozen & $\begin{array}{l}\text { Adenoma from: } \\
\text { FAP-patient } \\
\text { Multiple Adenoma patient } \\
\text { MSI-H CRC-Pat. } \\
\text { MSS/MSI-L CRC-Pat. }\end{array}$ & $\begin{array}{l}22 / 33 \\
12 / 29 \\
6 / 14 \\
8 / 15\end{array}$ & $\begin{array}{l}66 \\
41 \\
43 \\
53\end{array}$ & MSP & $\begin{array}{l}\text { Wynter et al. (2006) } \\
\text { [149] }\end{array}$ \\
\hline MINT31 & $\begin{array}{l}\text { Homo sapiens clone MINT31 } \\
\text { colon cancer differentially } \\
\text { methylated } \\
\text { CpG island genomic sequence. }\end{array}$ & $\begin{array}{l}\text { Endoscopic biopsy } \\
\text { specimen }\end{array}$ & $\begin{array}{l}\text { Serrated Adenomas } \\
\text { Tubular Adenomas } \\
\text { Normal Tissue }\end{array}$ & $\begin{array}{l}8 / 28 \\
8 / 34 \\
1 / 16\end{array}$ & $\begin{array}{l}29 \\
24 \\
6\end{array}$ & MSP & Park et al. (2003) [138] \\
\hline MLH1 & $\begin{array}{l}\text { MutL homolog 1, colon cancer, } \\
\text { nonpolyposis type } 2\end{array}$ & & $\begin{array}{l}25 \text { MSS CRC } \\
28 \text { MSI CRC } \\
\end{array}$ & $\begin{array}{l}0 / 25 \\
11 / 28 \\
\end{array}$ & \begin{tabular}{|l|}
0 \\
39 \\
\end{tabular} & MSP & Lind et al. (2004) [105] \\
\hline MLH1 & $\begin{array}{l}\text { MutL homolog } 1 \text {, colon cancer, } \\
\text { nonpolyposis type } 2\end{array}$ & $\begin{array}{l}\text { paraffin-embedded } \\
\text { and fresh frozen }\end{array}$ & HNPCC & $1 / 14$ & & MSP & Gazzoli et al. (2002) [152] \\
\hline MLH1 & $\begin{array}{l}\text { MutL homolog } 1 \text {, colon cancer, } \\
\text { nonpolyposis type } 2\end{array}$ & $\begin{array}{l}\text { Formalin-fixed, } \\
\text { paraffin-embedded }\end{array}$ & $\begin{array}{l}\text { MSS } \\
\text { MSI }\end{array}$ & $\begin{array}{l}18 / 33 \\
18 / 90\end{array}$ & $\begin{array}{l}55 \\
20 \\
\end{array}$ & COBRA & $\begin{array}{l}\text { Nakagawa et al. (2001) } \\
\text { [93] }\end{array}$ \\
\hline MLH1 & $\begin{array}{l}\text { MutL homolog } 1 \text {, colon cancer, } \\
\text { nonpolyposis type } 2\end{array}$ & $\begin{array}{l}\text { Endoscopic biopsy } \\
\text { specimen }\end{array}$ & $\begin{array}{l}\text { Serrated Adenomas } \\
\text { Tubular Adenomas }\end{array}$ & $\begin{array}{l}4 / 26 \\
0 / 34\end{array}$ & $\begin{array}{l}15 \\
0\end{array}$ & MSP & Park et al. (2003) [138] \\
\hline MLH1 & $\begin{array}{l}\text { MutL homolog 1, colon cancer, } \\
\text { nonpolyposis type } 2\end{array}$ & Fresh frozen & $\begin{array}{l}\text { IBD neoplasia } \\
\text { MSI-H } \\
\text { MSI-L } \\
\text { MSS }\end{array}$ & $\begin{array}{l}6 / 13 \\
1 / 16 \\
4 / 27\end{array}$ & \begin{tabular}{|l}
46 \\
6 \\
15
\end{tabular} & MSP & $\begin{array}{l}\text { Fleisher et al. (2000) } \\
\text { [153] }\end{array}$ \\
\hline $\begin{array}{l}\text { MYOD } \\
\text { (MYF-3) }\end{array}$ & myogenic differentiation 1 & Fresh frozen & $\begin{array}{l}\text { Adenomas } \\
\text { CRC }\end{array}$ & $\begin{array}{l}15 / 17 \\
104 / 105\end{array}$ & $\begin{array}{l}88 \\
99 \\
\end{array}$ & SB & $\begin{array}{l}\text { Shannon et al. (1999) } \\
\text { [154] }\end{array}$ \\
\hline PTGIS & $\begin{array}{l}\text { Prostaglandin I2 (prostacyclin) } \\
\text { synthase }\end{array}$ & Fresh frozen & $\begin{array}{l}\text { Adenoma } \\
\text { CRC }\end{array}$ & $\begin{array}{l}3 / 10 \\
43 / 100\end{array}$ & $\begin{array}{l}30 \\
43 \\
\end{array}$ & BS & Frigola et al. (2005) [130] \\
\hline $\begin{array}{l}\text { PTGS } \\
\text { (Cox-2) }\end{array}$ & \begin{tabular}{|l|} 
prostaglandin-endoperoxide \\
synthase 2 (Cyclooxygenase)
\end{tabular} & & $\begin{array}{l}\text { CRC } \\
\text { Adenoma }\end{array}$ & $\begin{array}{l}12 / 92 \\
7 / 50\end{array}$ & $\begin{array}{l}13 \\
14 \\
\end{array}$ & COBRA & Toyota et al. (2000) [131] \\
\hline RASSF1 & $\begin{array}{l}\text { Ras association (RalGDS/AF-6) } \\
\text { domain family } 1\end{array}$ & $\begin{array}{l}\text { Formalin-fixed, } \\
\text { paraffin-embedded }\end{array}$ & $\begin{array}{l}\text { CRC } \\
\text { Normal tissue HD } \\
\text { Normal tissue CRC-Pat }\end{array}$ & $\begin{array}{l}45 / 222 \\
0 / 6 \\
1 / 7\end{array}$ & $\begin{array}{l}20 \\
0\end{array}$ & MSP & $\begin{array}{l}\text { Van Engeland et al. } \\
\text { (2002) [155] }\end{array}$ \\
\hline RASSF1 & $\begin{array}{l}\text { Ras association (RalGDS/AF-6) } \\
\text { domain family } 1\end{array}$ & & $\begin{array}{l}\text { MSI+CRC } \\
\text { HNPCC }\end{array}$ & $\begin{array}{l}16 / 31 \\
6 / 20 \\
\end{array}$ & $\begin{array}{l}52 \\
30 \\
\end{array}$ & MSP & $\begin{array}{l}\text { Oliveira et al. (2005) } \\
\text { [156] }\end{array}$ \\
\hline RASSF2 & $\begin{array}{l}\text { Ras association (RalGDS/AF-6) } \\
\text { domain family } 2\end{array}$ & & $\begin{array}{l}\text { Adenoma } \\
\text { CRC }\end{array}$ & $\begin{array}{l}21 / 49 \\
51 / 122 \\
\end{array}$ & \begin{tabular}{|l|}
43 \\
42 \\
\end{tabular} & COBRA & $\begin{array}{l}\text { Akino et al. (2005) } \\
{[132]}\end{array}$ \\
\hline RUNX3 & runt-related transcription factor 3 & & CRC & $31 / 92$ & 34 & MSP & $\begin{array}{l}\text { Imamura et al. (2005) } \\
\text { [157] }\end{array}$ \\
\hline $\begin{array}{l}\text { SFRP1 } \\
\text { SFRP2 } \\
\text { SFRP4 } \\
\text { SFRP5 }\end{array}$ & $\begin{array}{l}\text { Secreted frizzled-related protein } \\
1,2,4,5\end{array}$ & & CRC & $\begin{array}{l}118 / 124 \\
111 / 124 \\
36 / 124 \\
73 / 124\end{array}$ & $\begin{array}{l}95 \\
90 \\
29 \\
59\end{array}$ & MSP & Suzuki et al. (2002) [108] \\
\hline SLC5A8 & $\begin{array}{l}\text { solute carrier family } 5 \text { (iodide } \\
\text { transporter), member } 8\end{array}$ & & $\begin{array}{l}\text { CRC } \\
\text { Normal tissue CRC-Pat } \\
\text { Normal tissue HD }\end{array}$ & $\begin{array}{l}38 / 64 \\
0 / 26 \\
0 / 12\end{array}$ & 59 & MSP & Li et al. (2003) [101] \\
\hline $\begin{array}{l}\text { SMARCA3 } \\
\text { (HLTF) }\end{array}$ & $\begin{array}{l}\text { SWI/SNF related, matrix } \\
\text { associated, actin dependent } \\
\text { regulator of chromatin, subfamily } \\
\text { a, member } 3\end{array}$ & & CRC & $27 / 63$ & 43 & MSP & $\begin{array}{l}\text { Moinova et al. (2002) } \\
{[134]}\end{array}$ \\
\hline $\begin{array}{l}\text { TMEFF2 } \\
\text { (TPEF/HPP1) }\end{array}$ & $\begin{array}{l}\text { transmembrane protein with EGF- } \\
\text { like and two follistatin-like } \\
\text { domains } 2\end{array}$ & Fresh tissue & $\begin{array}{l}\text { Normal tissue } \\
\text { CRC } \\
\text { Liver metastasis }\end{array}$ & $\begin{array}{l}1 / 21 \\
36 / 47 \\
19 / 24 \\
\end{array}$ & \begin{tabular}{|l|}
5 \\
77 \\
79 \\
\end{tabular} & $\mathrm{ML}$ & Ebert et al. (2005) [135] \\
\hline $\begin{array}{l}\text { TMEFF2 } \\
\text { (TPEF/HPP1) }\end{array}$ & $\begin{array}{l}\text { transmembrane protein with EGF- } \\
\text { like and two follistatin-like } \\
\text { domains } 2\end{array}$ & Fresh frozen & $\begin{array}{l}\text { Adenoma from: } \\
\text { FAP-patient } \\
\text { Multiple Adenoma patient } \\
\text { MSI-H CRC-Pat. } \\
\text { MSS/MSI-L CRC-Pat. } \\
\end{array}$ & $\begin{array}{l}10 / 32 \\
17 / 26 \\
9 / 14 \\
7 / 13\end{array}$ & $\begin{array}{l}31 \\
65 \\
64 \\
54 \\
\end{array}$ & COBRA & $\begin{array}{l}\text { Wynter et al. (2006) } \\
\text { [149] }\end{array}$ \\
\hline $\begin{array}{l}\text { TMEFF2 } \\
\text { (TPEF/HPP1) }\end{array}$ & $\begin{array}{l}\text { transmembrane protein with EGF- } \\
\text { like and two follistatin-like } \\
\text { domains } 2\end{array}$ & Fresh tissue & $\begin{array}{l}\text { Adenoma } \\
\text { HP } \\
\text { AC }\end{array}$ & $\begin{array}{l}6 / 9 \\
17 / 27 \\
46 / 55 \\
\end{array}$ & \begin{tabular}{|l|}
66 \\
63 \\
84 \\
\end{tabular} & COBRA & Young et al. (2001) [136] \\
\hline $\begin{array}{l}\text { TMEFF2 } \\
\text { (TPEF/HPP1) }\end{array}$ & $\begin{array}{l}\text { transmembrane protein with EGF- } \\
\text { like and two follistatin-like } \\
\text { domains } 2\end{array}$ & $\begin{array}{l}\text { Formalin-fixed, } \\
\text { paraffin-embedded }\end{array}$ & $\begin{array}{l}\text { UC mucosa } \\
\text { Dysplasia } \\
\text { CRC }\end{array}$ & $\begin{array}{l}0 / 5 \\
4 / 10 \\
24 / 48\end{array}$ & $\begin{array}{l}0 \\
40 \\
50\end{array}$ & MSP & Sato et al. (2002) [87] \\
\hline VIM & Vimentin & Fresh tissue & $\begin{array}{l}\text { CRC patients } \\
\text { Healthy Donor }\end{array}$ & $\begin{array}{l}95 / 153 \\
1 / 46\end{array}$ & $\begin{array}{l}62 \\
2\end{array}$ & MSP & Chen et al. (2005) [158] \\
\hline
\end{tabular}


Table 2c

DNA methylation in serum/plasma and stool of CRC patients

\begin{tabular}{|c|c|c|c|c|c|c|c|}
\hline Gene & Name & Specimen & Donor & $\begin{array}{l}\text { Fraction } \\
\text { methylated }\end{array}$ & $\begin{array}{l}\text { Percentage } \\
\text { methylated }\end{array}$ & $\begin{array}{l}\text { Technique } \\
\text { used }\end{array}$ & Reference \\
\hline $\mathrm{CDH} 4$ & Cadherin 4, R-cadherin & Peripheral blood & $\begin{array}{l}\text { CRC patient } \\
\text { Blood lymphocytes }\end{array}$ & $\begin{array}{l}32 / 46 \\
0 / 17\end{array}$ & $\begin{array}{l}70 \\
0\end{array}$ & MSP & $\begin{array}{l}\text { Miotto } \text { et al. (2004) } \\
{[119]}\end{array}$ \\
\hline $\begin{array}{l}\text { CDKN2A } \\
\text { (P16) }\end{array}$ & cyclin-dependent kinase inhibitor $2 \mathrm{~A}$ & Stool & $\begin{array}{l}\text { Patients with adenomas } \\
\text { Healthy Donor }\end{array}$ & $\begin{array}{l}9 / 29 \\
3 / 19\end{array}$ & $\begin{array}{l}31 \\
16\end{array}$ & MSP & Petko et al. (2005) [96] \\
\hline $\begin{array}{l}\text { CDNK2A } \\
\text { (P16) }\end{array}$ & cyclin-dependent kinase inhibitor $2 \mathrm{~A}$ & 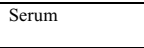 & CRC patients & $13 / 44$ & 30 & MSP & $\begin{array}{l}\text { Nakayama et al. (2002) } \\
{[113]}\end{array}$ \\
\hline $\begin{array}{l}\text { CDNK2A } \\
\text { (P16) }\end{array}$ & cyclin-dependent kinase inhibitor $2 \mathrm{~A}$ & Serum & $\begin{array}{l}\text { CRC patients with } \\
\text { recurrence }\end{array}$ & $31 / 45$ & 69 & MSP & $\begin{array}{l}\text { Nakayama et al. (2003) } \\
{[148]}\end{array}$ \\
\hline $\begin{array}{l}\text { CDNK2A } \\
\text { (P16) }\end{array}$ & cyclin-dependent kinase inhibitor $2 \mathrm{~A}$ & Serum & \begin{tabular}{|l} 
CRC patients \\
Patients with Adenomas \\
Healthy Individuals \\
\end{tabular} & $\begin{array}{l}14 / 20 \\
0 / 34 \\
0 / 10 \\
\end{array}$ & 70 & MSP & Zou et al. (2002) [114] \\
\hline $\begin{array}{l}\text { CDNK2A } \\
\text { (P16) }\end{array}$ & cyclin-dependent kinase inhibitor $2 \mathrm{~A}$ & Plasma & CRC patients & $21 / 31$ & 68 & MSP & $\begin{array}{l}\text { Lecomte } \text { et al. (2002) } \\
{[115]}\end{array}$ \\
\hline DAPK1 & Death associated protein kinase 1 & Serum & CRC patients & $3 / 14$ & 21 & MSP & $\begin{array}{l}\text { Yamaguchi } \text { et al. (2003) } \\
{[150]}\end{array}$ \\
\hline $\mathrm{HICl}$ & Hypermethylated in cancer 1 & Stool & $\begin{array}{l}\text { CRC patients } \\
\text { Patients with Adenomas } \\
\text { Patients with HP } \\
\text { Healthy Individuals }\end{array}$ & & \begin{tabular}{|l|}
42 \\
31 \\
0 \\
0
\end{tabular} & MSP & $\begin{array}{l}\text { Lenhard et al. (2005) } \\
\text { [159] }\end{array}$ \\
\hline MGMT & $\begin{array}{l}\text { O-6-methylguanine-DNA } \\
\text { methyltransferase }\end{array}$ & Stool & $\begin{array}{l}\text { Patients with adenomas } \\
\text { Healthy Donor }\end{array}$ & $\begin{array}{l}14 / 29 \\
5 / 18\end{array}$ & $\begin{array}{l}48 \\
27\end{array}$ & MSP & Petko et al. (2005) [96] \\
\hline MLH1 & $\begin{array}{l}\text { MutL homolog } 1 \text {, colon cancer, } \\
\text { nonpolyposis type } 2\end{array}$ & Serum & $\begin{array}{l}\text { Matched serum samples to } \\
\text { MLH positive CRCs }\end{array}$ & $3 / 9$ & 33 & & Grady et al. (2001) [112] \\
\hline MLH1 & $\begin{array}{l}\text { MutL homolog } 1 \text {, colon cancer, } \\
\text { nonpolyposis type } 2\end{array}$ & Serum & $\begin{array}{l}\text { CRC patients } \\
\text { Healthy Donor }\end{array}$ & $\begin{array}{l}19 / 49 \\
1 / 41\end{array}$ & $\begin{array}{l}39 \\
2\end{array}$ & MSP & Leung et al. (2005) [160] \\
\hline PGR & progesterone receptor & Stool & $\begin{array}{l}\text { CRC patients } \\
\text { Healthy Donor }\end{array}$ & $\begin{array}{l}18 / 23 \\
8 / 26\end{array}$ & $\begin{array}{l}78 \\
31\end{array}$ & ML & Müller et al. (2004) [38] \\
\hline SFRP2 & Secreted frizzled-related protein 2 & Stool & $\begin{array}{l}\text { CRC patients } \\
\text { Healthy Donor }\end{array}$ & $\begin{array}{l}19 / 23 \\
6 / 26\end{array}$ & $\begin{array}{l}83 \\
26\end{array}$ & ML & Müller et al. (2004) [38] \\
\hline SFRP5 & Secreted frizzled-related protein 5 & Stool & $\begin{array}{l}\text { CRC patients } \\
\text { Healthy Donor }\end{array}$ & $\begin{array}{l}18 / 23 \\
9 / 26\end{array}$ & $\begin{array}{l}78 \\
35 \\
\end{array}$ & ML & Müller et al. (2004) [38] \\
\hline $\begin{array}{l}\text { SMARCA3 } \\
\text { (HLTF) }\end{array}$ & $\begin{array}{l}\text { SWI/SNF related, matrix associated, } \\
\text { actin dependent regulator of } \\
\text { chromatin, subfamily a, member } 3\end{array}$ & Serum & $\begin{array}{l}\text { CRC patients } \\
\text { Healthy Donor }\end{array}$ & $\begin{array}{l}14 / 49 \\
3 / 41\end{array}$ & $\begin{array}{l}29 \\
7\end{array}$ & MSP & Leung et al. (2005) [160] \\
\hline VIM & Vimentin & Stool & $\begin{array}{l}\text { CRC patients } \\
\text { Healthy Donor }\end{array}$ & $\begin{array}{l}43 / 94 \\
20 / 198\end{array}$ & $\begin{array}{l}46 \\
10 \\
\end{array}$ & MSP & Chen et al. (2005) [158] \\
\hline
\end{tabular}

\begin{tabular}{|c|c|}
\hline$-\mathrm{CL}$ & Colorectal cancer cell lines \\
\hline CRC-Pat & Colorectal cancer patient \\
\hline HD & Healthy donor \\
\hline HP & Hyperplastic polyp \\
\hline HM & Hypermethylation \\
\hline FAP & Familial Adenomatous Polyposis \\
\hline $\mathrm{UC}$ & Ulcerative Colitis \\
\hline IBD & Inflammatory Bowel disease neoplasia \\
\hline HNPCC & hereditary nonpolyposis colon cancer \\
\hline MSI-H & Microsatellite Instability high-frequency \\
\hline MSI-L & Microsatellite Instability low-frequency \\
\hline MSS & Microsatellite stable \\
\hline CIMP+ & $\mathrm{CpG}$ island methylator phenotype positive \\
\hline CIMP- & $\mathrm{CpG}$ island methylator phenotype negative \\
\hline
\end{tabular}

early colorectal carcinogenesis are e.g. SMAD2/4, TP53 (for review see [23]).

As outlined above, the Wingless/Wnt signaling pathway plays a vital role in malignant transformation during the adenoma-carcinoma sequence [55]. Aberrant WNT signaling is an early event in the process of carcinogenesis in approximately $90 \%$ of CRCs [70]. It occurs mainly through inactivating mutations of the tumor suppressor gene APC [71-73] and less often through mutations of $\beta$-catenin or AXIN2 [74,75]. These alterations result in cellular accumulation of $\beta$-catenin, which subsequently serves as an activator of T-cell factor/lymphoid-enhancing factor (Tcf/LEF)dependent transcription. Several $\beta$-catenin/TCF target genes are presumed to contribute to tumor initiation and progression in mice and humans [76].

\subsection{DNA methylation in general}

Changes in the status of DNA methylation, known as epigenetic alterations, are one of the most common molecular alterations in human neoplasia [77]. Epigenetic changes differ from genetic changes mainly in that they occur at a higher frequency than do genetic changes, are reversible upon treatment with pharmacological agents and occur at defined regions in a gene. Epigenetics describes a trait that is heritable, yet not based on a change in primary DNA sequence [77-79]. In recent years it has become clear that there is a synergy between genetic and epigenetic changes and that Knudson's two-hit hypothesis needs to be revised: instead of only two possibilities (loss of heterozygosity or homozygous deletion), there is also a third possibility - transcriptional silencing by DNA methylation 
of promoters - that can disable tumor-suppressor gene transcription [80].

Cytosine methylation occurs after DNA synthesis, by enzymatic transfer of a methyl group from the methyl donor S-adenosylmethionine to the carbon-5 position of cytosine. Cytosines are methylated in the human genome mostly when located 5 ' to a guanosine. In human somatic cells, $5 \mathrm{mC}$ accounts for $\sim 1 \%$ of total DNA bases and therefore affects $70 \%-80 \%$ of all $\mathrm{CpG}$ dinucleotides in the genome. These $\mathrm{CpG}$ dinucleotides are severely under-represented in the human genome, because they are affected by a high rate of methylcytosine-to-thymine transition mutations. Remaining CpG dinucleotides are unequally distributed across the human genome, which means there exist stretches of sequences without $\mathrm{CpG}$ dinucleotides interspersed by so-called $\mathrm{CpG}$ islands. $\mathrm{CpG}$ islands are defined as a 500-base-pair window with a G:C content of at least $55 \%$ and an observed overexpected frequency of at least 0.65 . Computational analysis of the human genome sequence predicts $29000 \mathrm{CpG}$ islands. It has been increasingly recognized over the past years that the $\mathrm{CpG}$ islands of a large number of genes, which are mostly unmethylated in normal tissue, are methylated to varying degrees in human cancers. Methylation of some $\mathrm{CpG}$ islands in non-malignant tissue also increases with age, whereas the total genomic content of $5 \mathrm{mC}$ declines. The same is true during carcinogenesis of several tumors (e.g. adenoma-carcinoma sequence), where methylation takes place at specific promoter regions, followed by general hypomethylation of the whole genome, and this is thought to induce a higher rate of chromosomal instability (for review see [7779,81]). Post-synthetic covalent addition of a methyl group to cytosine is mediated by the three known active DNA cytosine methyltransferases (DNMT1, 3a, and 3b). When DNA containing a symmetrically methylated $\mathrm{CpG}$ dinucleotide is replicated, the result is two double-stranded DNA molecules, each containing a methylated $\mathrm{CpG}$ dinucleotide on the parental strand, but also containing an unmethylated $\mathrm{CpG}$ dinucleotide on the newly synthesized strand. The methylated state of the site in the parent molecule is maintained in the daughter molecules when a maintenance methyltransferase recognizes the hemimethylated site and methylates the unmethylated cytosine, restoring the symmetrically methylated $\mathrm{CpG}$ dinucleotide pair. DNMT1 is mainly responsible for maintenance of DNA methylation, whereas DNMT3a and DNMT3b have been shown to methylate hemimethylated and unmethylated DNA with equal efficiency. Overexpression of both DNMT1 and DNMT3 mRNAs has been reported in human tumors.

The reciprocal relationship between the density of methylated cytosine residues and the transcriptional activity of a gene has been widely documented. It should be emphasized, however, that this inverse correlation has been demonstrated conclusively only for methylation in the promoter regions and not in the transcribed parts of a gene. Several tumor-suppressor genes contain $\mathrm{CpG}$ islands in their promoters, and many of them show evidence of methylation silencing. After changes associated with histone deacetylation have occurred and these $\mathrm{CpG}$ islands have become methylated, the relevant genes become irrevocably silent (for review see [77-79,81]).

Advances in the technology of DNA methylation analysis have spurred the discovery of numerous cases of hypermethylation of tumor-suppressor gene promoters in human tumors. Furthermore, it has become clear that methylated DNA can be detected in tumor-derived DNA found in the serum of cancer patients [47-52]. Additionally, methylated DNA can also be found in samples obtained from cancer patients by draining to the outside of the body either physiologically (stool, vaginal secretion $[38,82]$ ) or artificially (peritoneal fluids [83]).

\subsection{Adenoma-carcinoma sequence-epigenetic alterations}

The past decade saw a large number of studies dealing with DNA methylation changes in tumorigenesis of CRC. The data concerning hypermethylation were predated by studies of global hypomethylation at an early stage in colorectal neoplasia (for review see [84]). Age is the principal function of CRC incidence, and age-related methylation changes are well documented for CRC [84]. Another risk factor for CRC is ulcerative colitis (UC). Interestingly, it was found that both the dysplastic and nondysplastic mucosa of UC patients with neoplasia have significantly elevated levels of agerelated methylation, indicating that chronic inflammation is associated with high levels of methylation, perhaps as a result of increased cell turnover, and that UC can be viewed as resulting in premature aging of colorectal epithelial cells [85]. Furthermore, specific hypermethylation was also seen to be a very early event in UC-associated carcinogenesis, thus indicating the possibility that hypermethylation can serve as a biomarker for early detection of cancer or dysplasia in UC [86, 87]. 
Deficiencies in the mismatch repair (MMR) system result in mutation rates 100 -fold greater than for normal cells as a direct consequence of an inability to faithfully replicate the genome. In particular, these mutations are evident as frameshifts in microsatellite sequences. They are normally stable, but slippage during DNA replication generates insertions/deletions and, if perpetuated, engenders microsatellite instability (MSI), the hallmark of the replication error phenotype. MSI is also present in $10 \%-15 \%$ of cases of sporadic colorectal cancer, but is rarely caused by mutation. It has been reported that a strong correlation exists between genetic instability and methylation capacity, indicating that methylation abnormalities may play a role in chromosome segregation in cancer cells. A central MMR gene, called $M L H 1$, was reported to be methylated in sporadic CRCs and strongly associated with MSI [84,88-90]. The finding of aberrant methylation of $M L H 1$ in sporadic MSI colon cancers, and the restoration of MLH1 expression by demethylating the MLH1 promoter in cell lines derived from such cancers, strongly suggests that such aberrant methylation could be a cause rather than a consequence of colon carcinogenesis [84,88-90]. Fine-structure analysis of the methylation status of specific CpGs in the $M L H 1$ promoter has shown that the methylation status of small clusters of CpGs in the 5' region of the MLHI promoter appears to dictate the transcriptional status of the gene [91]. Hawkins and Ward [92] also reported such a $M L H 1$ hypermethylation in hyperplastic polyps of patients with sporadic CRC with MSI, suggesting that hypermethylation of the $M L H 1$ gene is a critical step in progression to carcinoma. It was recently shown that methylation of $M L H 1$ promoter in the normal colonic mucosa is closely associated with age and the development of sporadic MSI+ colon cancers [93].

As mentioned above, several findings gave rise to the hypothesis that epigenetic and genetic changes act together to promote cancer formation [94]. Although mutation of $C D K N 2 A / p 16$ has not been described in CRC, methylation of $C D K N 2 A / p 16$ is detected in $40 \%$ of CRCs [95]. Furthermore, it has been reported that methylation plays an important role in colon adenomas [96,97]. For example, $C D K N 2 A / p 16$ methylation is more common in adenomas with tubulovillous/villous histology, a characteristic associated with more frequent predisposition to invasive carcinoma [97]. This observation demonstrates that aberrant promoter methylation occurrs early in the adenoma carcinoma sequence, although it does not confirm that the aberrant $C D K N 2 A / p 16$ methylation is a primary, rather than a secondary, event in the tumorigenesis process. Additionally, DNA methylation status of $M G M T, C D K N 2 A$, and $M L H 1$ in colon adenomas and hyperplastic polyps has been determined to evaluate the timing and frequency of these events in the adenoma-carcinoma progression sequence and subsequently to analyze the potential for these methylated genes to be molecular markers for adenomas and hyperplastic polyps [96]. It has been revealed that methylated MGMT, CDKN2A and MLHI occur in 49\%, 34\% and $7 \%$ of adenomas and in $5 \%, 10 \%$ and $7 \%$ of hyperplastic polyps, respectively, and that they are more common in histologically advanced adenomas. Furthermore, methylated $C D K N 2 A, M G M T$ and $M L H 1$ were detected in fecal DNA from $31 \%, 48 \%$ and $0 \%$ of individuals with adenomas, indicating the potential of fecal DNA-based assays as a useful diagnostic test for polyps [96].

In addition to the interest in the role of epigenetic alterations in established cancers, the evidence showing increased methylation in $\mathrm{CpG}$ islands in non-neoplastic tissues has prompted considerable interest in the role aberrant DNA methylation may have as a pre-neoplastic event. Indeed, there is evidence that aberrant $\mathrm{CpG}$ island methylation may occur as the result of a genetic predisposition or a field effect. Ahuja et al. [98] showed that aberrant $\mathrm{CpG}$ island methylation occurs in histologically normal colon epithelium in an age-dependent fashion and that half of the genes involved in this agerelated methylation are the same as those involved in colon carcinogenesis. The cause of this age-related DNA methylation is unknown, but current models suggest that the methylation occurs as the consequence of local predisposing factors in DNA (e.g. methylation control centers, such as $\mathrm{Sp} 1$ (specificity protein 1) sites or tandem B1 elements), environmental exposures, and/or a genetic predisposition to aberrant DNA methylation (for review see [55]). Furthermore, it is likely detection of colon adenomas with methylation may identify colonic epithelium that is at significant risk for genetic alterations that will lead to colon tumor formation [99].

As mentioned above, the adenoma-carcinoma progression is believed to be an evolutionary process in which neoplastic cells acquire heritable genetic and epigenetic alterations that drive the carcinogenesis process. Each major step in this evolutionary process is usually accompanied by a recognizable histological change that proceeds from a benign tubular adenoma to an advanced adenoma (e.g. tubulovillous or villous adenoma) and finally to invasive adenocarcinoma. It 
is supposed for CRC that specific gene mutations (e.g. $A P C$ mutations) initiate the formation of tubular adenomas and that others (e.g. TP53 mutations) drive the malignant transformation of the adenomas (for review see [55]).

Aberrant DNA methylation of specific loci has been identified in the earliest precursor lesions for colon adenocarcinomas, aberrant crypt foci (ACF). MINT1, MINT31, SLC5A8 and MGMT methylation has been found in ACFs and in adenomas [97,100,101]. Additionally, early work has reported that CRCs with hypermethylated $M L H 1$ and/or $C D K N 2 A / p 16$ may belong to a distinct subclass of CRCs, termed the CIMP (CpG island methylator phenotype), that demonstrate genomewide aberrant methylation of gene promoters and that may be caused by a distinct and unique mechanism. Interestingly, the specific genes commonly found to be methylated in CRC differ from those commonly found to be methylated in other tumor types, suggesting that there is a selective process driving the occurrence of methylated genes [95,102].

Bai et al. [103] found that the methylation status of genes is established in the adenoma phase of the adenoma-carcinoma sequence, suggesting that these events occur during initiation of the colon neoplasms and do not have a functional role in the progression of colon cancer. In contrast, it has been observed that a subset of genes (MLH1, RASSF1, CDKN2A, GSTP1, THBS1 and TIMP3) was more commonly methylated in CRCs than in adenomas, suggesting that at least some genes may affect the transformation step in CRC formation [104]. The same study group found no difference in the proportion of genes methylated in progressively more advanced stages of adenocarcinoma, but they did not assess the frequencies of specific methylated genes in various stages of CRC, which would be more informative with regard to understanding the role of epigenetic events in the clonal evolution of CRC [104].

Furthermore, recent studies have shown that epigenetic silencing of genes involved in Wingless/Wnt signaling is an alternative mechanism in colorectal carcinogenesis. Compared to inactivating $A P C$ mutations, epigenetic silencing of $A P C$ seems to play a minor role [105]. Koinuma et al. [106] documented epigenetic silencing of AXIN2 in MSI+ colorectal cancer. Nevertheless, two groups have documented frequent promotor hypermethylation and epigenetic silencing of genes encoding secreted frizzled-related proteins (SFRPs) which are thought to contribute to constitutive WNT signaling $[107,108]$. While SFRP and WIF-1 [109] methylation-associated silencing occurs across the whole spectrum of colorectal tumorigenesis, Aguilera et al. [110] demonstrated that Dickkopf-1 $(D K K-1)$ promotor hypermethylation was present only in advanced colorectal neoplasms.

At bottom, significant evidence is provided that the aberrant methylation of genes contributes to the initiation of adenomas and their progression to CRC. A summary of reported methylated genes in CRC cell lines or CRC tissue is presented in Table $2 \mathrm{a}$ and $2 \mathrm{~b}$.

\subsection{Epigenetic alterations in the bloodstream of CRC patients}

An increasing number of studies have reported the presence of methylated DNA in serum/plasma of patients with various types of malignancies and the absence of methylated DNA in normal control patients (for review see [47]). For the past five years our research group was mainly interested in evaluating DNA methylation changes in serum of cancer patients [4752 ]. We came to the conclusion that there is great potential for the use of these epigenetic markers as early detection markers, markers for prognostication and even for therapy monitoring. In terms of CRC, several interesting studies have been reported: Two decades ago, Shapiro et al. [111] for the first time reported markedly elevated circulating DNA levels in patients with malignant gastrointestinal disease as compared to moderately elevated levels in benign disease and minimal values in normal controls. Grady et al. [112] found methylated $M L H 1$ promoter DNA in the serum of patients with microsatellite unstable CRCs. Other studies reported aberrant $p 16$ methylation in the serum of CRC patients, indicating its potential role as a tumor marker [113,114]. Methylated $p 16$ tumor DNA in the serum or plasma of CRC patients seems to be associated with later Dukes' stages and with poorer prognosis [114,115]. A summary of reported methylated genes in serum/plasma of CRC patients is presented in Table 2c. The potential use of circulating methylated DNA in serum/plasma of CRC patients for therapy monitoring is presented in Fig. 1.

\subsection{Epigenetic alterations in stool of CRC patients}

Detection of hypermethylated DNA markers might help identify patients with CRC and precursors using stool. An initial feasibility study using a panel of three markers (CDKN2A, MGMT and MLH1) detected hypermethylation in DNA extracted from stools of seven of 12 CRC patients (giving a sensitivity of 58\%), but 
also in three of ten normal controls (giving a specificity of $70 \%$ ) (for review see [23]).

Very recently, our study group [38] was able to demonstrate secreted frizzled-related protein 2 (SFRP2) methylation - an antagonist of the WNT signaling pathway that is commonly methylated in CRC tissue specimens [108] - as the most sensitive single DNA-based marker in stool for identification of CRC (sensitivity 77\%-90\%, specificity 77\%). For this study we used MethyLight analysis of fecal DNA from three independent sets of patients. Additionally, DNA methylation was detected in three of five proximal (right-sided) cancers.

Furthermore, Petko et al. [96] demonstrated that detection of methylated genes in fecal DNA from individuals with colon polyps carrying methylated genes is possible. Detection of DNA methylation in fecal DNA holds promise as a key component of screening modalities for CRC, not least of all in view of its potential contribution to detecting proximal (right-sided) cancers (Fig. 1). It remains to be seen whether a combination of genetic and epigenetic markers will identify CRC at an early stage. A summary of reported methylated genes in stool of CRC patients is presented in Table $2 \mathrm{c}$.

Consequently, detection of genetic or epigenetic alteration or both in several specimens (stool, blood) from patients with CRC may have the potential for early detection of CRC. Effective early detection of adenomas would offer several benefits, such as lowered CRC incidence and reduced need for surgical intervention. As a lengthy period is required for CRC to develop from an adenoma, an effective adenoma screening test would need to be performed less frequently than a test for early CRC. However, because only a small minority of adenomas is destined to progress to malignancy, detection of adenomas would involve gross overtreatment of patients, which would be costly and harmful, both physically and psychologically. It could be argued that the optimal test would be one that accurately detected advanced adenomas with a high chance of malignant progression, but this requires better understanding of the natural history of such lesions [23]. Genetic and epigenetic markers may also serve as tools for therapy monitoring in cancer patients in order to detect early progression of the disease and offer immediate and specific treatment regimens (e.g. curative resection of liver and lung metastases) as a means of ultimately ensuring longer survival and better quality of life.

\section{Conclusions}

CRC is a common malignancy. Advances in cancer treatment and improvements in cancer outcome over the past few decades have been modest. In the United States, $57 \%$ of CRC patients have regional or distant spread of their disease at the time of diagnosis [14]. Only modest gains in the survival of CRC patients with advanced disease at time of diagnosis have been achieved over the past few decades. Early detection seems to be one of the most important approaches to reducing mortality by identifying cancer while still localized and curable, as well as to reducing morbidity and costs. Furthermore, longer survival and better quality of life can be achieved with earlier detection of progressive CRC.

Cervical cancer (CC) provides an excellent example of the power of early detection. Its effectiveness is mostly due to: its high acceptance in the population; the fact that the "organ of interest" is easily accessible in a non-invasive procedure; detailed knowledge of the alterations during pathogenesis (progressing from lowgrade to intermediate- to high-grade CIN and eventually to invasive cancer); and that changes can easily be identified by a very well-established marker like cytology.

With regard to CRC, the natural history of the disease also seems to show progression from low-grade to intermediate- to high-grade lesions and eventually to invasive cancer (adenoma-carcinoma model of CRC carcinogenesis $[7,54,56])$. Especially alterations in the WNT pathway occur in malignant transformation during the adenoma-carcinoma sequence. Aberrant WNT signaling is an early event in the process of carcinogenesis in approximately $90 \%$ of CRCs [70]. Consequently, many of the genetically or epigenetically altered genes occurring during colorectal carcinogenesis (e.g. in the WNT pathway) may have some potential to serve as early detection markers or markers for therapy monitoring.

Early-detection researchers should therefore try to gain more insight into the molecular alterations occuring during progression from adenoma to carcinoma and should try to evaluate all possible target genes for early detection or therapy monitoring in CRC (as we attempted for DNA methylation markers in this review). Sullivan Pepe et al. [15] reported five phases of biomarker development for early detection of cancer. Up to now, research for early detection and therapy monitoring markers in CRC is still in Phase 1 and Phase 2 of the reported five phases: many preclinical ex- 
ploratory studies aiming to evaluate the expression and regulation of several genes (by mutation or epigenetic alteration) and proteins in CRC tissues and cell lines and comparable healthy organ tissue to identify candidates for early detection. Phase 2 of early-detection research still takes the shape of assay development and validation in specimens obtained non-invasively, such as serum, plasma or stool. The goal of these studies is to evaluate their ability to differentiate between patients with clinically established disease and healthy controls. To our knowledge, nothing has yet been published on the recommended phases 3, 4 and 5, including multicenter prospective screening studies and estimation of expected screening costs.

Finally, it must also be mentioned that the power of a screening test is not only dependent on its specificity and sensitivity but also on people's willingness to participate in a given screening program. Looking at CRC screening, only $40 \%$ of American women and men aged 50 years and older reported recent screening with an endoscopic procedure and only $20 \%$ of both sexes reported having undergone screening with a fecal occult blood test [12]. Reasons for such a small number of participants may be uncomfortable and unpleasant preparation procedures for endoscopy, sometimes painful examination procedures, complications during endoscopy and low sensitivity or specificity of FOBT.

Changes in DNA methylation have been recognized as one of the most common molecular alterations in human tumors, including CRC (for review see [84]). It has become clear that methylated DNA can be detected in tumor-derived DNA found in the bloodstream of cancer patients [47-52] and in samples obtained from cancer patients by draining to the outside of the body (stool, vaginal secretion $[38,82]$ or peritoneal fluids [83]). This specifically altered DNA may serve, on the one hand, as a possible new screening marker for CRC and, on the other hand, as a tool for therapy monitoring in patients having had CRC.

Very recently, our study group was able to identify secreted frizzled-related protein 2 (SFRP2) methylation in stool as the most sensitive single DNA-based marker for identification of CRC [38]. Additionally, detection of DNA methylation was successfully achieved in three of five proximal (right-sided) cancers. Therefore, testing for methylated DNA in stool samples may have great potential as an alternative screening tool for CRC (Fig. 1). As well as being of potential value in population screening, an effective molecular stool test might also be of use in reducing the frequency of follow-up surveillance colonoscopies required for patients with known disease (e.g. CRC in own history, long-standing inflammatory bowel disease with the known increased risk for CRC development; for review see [23]).

CRC recurrent disease mainly occurs in the regional abdominal lymph nodes, the liver or the lung and less frequently in the resected segment of the colorectum. Nevertheless, the life-threatening event in CRC is not lymph node metastasis per se, but hematogenous metastases which mainly affect the liver or the lung. Therefore, a screening test that is sensitive for hematogenous metastases and could be performed in patients' serum or plasma will have impact on early detection of patients with progressive CRC (Fig. 1). Such a test subsequently offers immediate start of specific treatment regimens (e.g. curative resection of liver and lung metastases).

Finally, we conclude that DNA methylation changes in CRC patients may serve, on the one hand, as a possible new screening marker for CRC and, on the other hand, as a tool for therapy monitoring in patients having had CRC.

\section{Acknowledgments}

This work was supported by a grant from "Jubiläumsfonds der Österreichischen Nationalbank"; Project \#10929, from "Tiroler Wissenschaftsfonds" and from "Österreichische Krebshilfe - Krebsgesellschaft Tirol".
Abbreviations

$\begin{array}{ll}\text { ACF } & \text { aberrant crypt focus } \\ \text { CC } & \text { cervical cancer } \\ \text { CRC } & \text { colorectal cancer } \\ \text { DCBE } & \text { double-contrast barium enema } \\ \text { FOBT } & \text { fecal occult blood test } \\ \text { FS } & \text { flexible sigmoidoscopy } \\ \text { MMR } & \text { mismatch repair } \\ \text { MSI } & \text { microsatellite instability } \\ \text { UC } & \text { ulcerative colitis }\end{array}$

\section{References}

[1] M. Shike, S.J. Winawer, P.H. Greenwald, A. Bloch, M.J. Hill and S.V. Swaroop, Primary prevention of colorectal cancer. The WHO Collaborating Centre for the Prevention of Colorectal Cancer, Bull World Health Organ 68 (1990), 377385 . 
[2] D.M. Parkin, Global cancer statistics in the year 2000, Lancet Oncol 2 (2001), 533-543.

[3] A. Jemal, T. Murray, E. Ward, A. Samuels, R.C. Tiwari, A Ghafoor, E.J. Feuer and M.J. Thun, Cancer statistics, 2005, CA Cancer J Clin 55 (2005), 10-30.

[4] A. Jemal, A. Thomas, T. Murray and M. Thun, Cancer statistics, 2002, CA Cancer J Clin 52 (2002), 23-47.

[5] J.E. Krook, C.G. Moertel, L.L. Gunderson, H.S. Wieand, R.T. Collins, R.W. Beart, T.P. Kubista, M.A. Poon, W.C. Meyers and J.A. Mailliard, Effective surgical adjuvant therapy for high-risk rectal carcinoma, N Engl J Med 324 (1991), 709-715.

[6] W. Willett, The search for the causes of breast and colon cancer, Nature 338 (1989), 389-394.

[7] E.R. Fearon and B. Vogelstein, A genetic model for colorectal tumorigenesis, Cell 61 (1990), 759-767.

[8] B. Reddy, A. Engle, S. Katsifis, B. Simi, H.P. Bartram, P. Perrino and C. Mahan, Biochemical epidemiology of colon cancer: effect of types of dietary fiber on fecal mutagens, acid, and neutral sterols in healthy subjects, Cancer Res 49 (1989), 4629-4635.

[9] J.D. Potter, Reconciling the epidemiology, physiology, and molecular biology of colon cancer, JAMA 268 (1992), 15731577.

[10] S. Winawer, R. Fletcher, D. Rex, J. Bond, R. Burt, J. Ferrucci, T. Ganiats, T. Levin, S. Woolf, D. Johnson, L. Kirk, S. Litin and C. Simmang, Colorectal cancer screening and surveillance: clinical guidelines and rationale-Update based on new evidence, Gastroenterology 124 (2003), 544-560.

[11] J.M. Walsh and J.P. Terdiman, Colorectal cancer screening: clinical applications, JAMA 289 (2003), 1297-1302.

[12] R.A. Smith, V. Cokkinides and H.J. Eyre, American Cancer Society guidelines for the early detection of cancer, 2004 CA Cancer J Clin 54 (2004), 41-52.

[13] D. Hayne, R.S. Brown, M. McCormack, M.J. Quinn, H.A Payne and P. Babb, Current trends in colorectal cancer: site, incidence, mortality and survival in England and Wales, Clin Oncol ( $R$ Coll Radiol) 13 (2001), 448-452.

[14] R. Etzioni, N. Urban, S. Ramsey, M. McIntosh, S. Schwartz, B. Reid, J. Radich, G. Anderson and L. Hartwell, Early detection: The case for early detection, Nat Rev Cancer 3 (2003), 243-252.

[15] P.M. Sullivan, R. Etzioni, Z. Feng, J.D. Potter, M.L. Thompson, M. Thornquist, M. Winget and Y. Yasui, Phases of biomarker development for early detection of cancer, $J$ Natl Cancer Inst 93 (2001), 1054-1061.

[16] W. Hamilton, A. Round, D. Sharp and T.J. Peters, Clinical features of colorectal cancer before diagnosis: a populationbased case-control study, Br J Cancer 93 (2005), 399-405.

[17] C.S. Huang, S.K. Lal and F.A. Farraye, Colorectal cancer screening in average risk individuals, Cancer Causes Control 16 (2005), 171-188.

[18] D.A. Ahlquist, D.B. McGill, S. Schwartz, W.F. Taylor and R.A. Owen, Fecal blood levels in health and disease. A study using HemoQuant, N Engl J Med 312 (1985), 1422-1428.

[19] J.D. Hardcastle, J.O. Chamberlain, M.H. Robinson, S.M. Moss, S.S. Amar, T.W. Balfour, P.D. James and C.M. Mangham, Randomised controlled trial of faecal-occult-blood screening for colorectal cancer, Lancet 348 (1996), 14721477.

[20] O. Kronborg, C. Fenger, J. Olsen, O.D. Jorgensen and O. Sondergaard, Randomised study of screening for colorectal cancer with faecal-occult-blood test, Lancet 348 (1996), 1467-1471
[21] J.S. Mandel, J.H. Bond, T.R. Church, D.C. Snover, G.M. Bradley, L.M. Schuman and F. Ederer, Reducing mortality from colorectal cancer by screening for fecal occult blood. Minnesota Colon Cancer Control Study, $N$ Engl J Med 328 (1993), 1365-1371.

[22] J.S. Mandel, T.R. Church, F. Ederer and J.H. Bond, Colorectal cancer mortality: effectiveness of biennial screening for fecal occult blood, J Natl Cancer Inst 91 (1999), 434-437.

[23] R.J. Davies, R. Miller and N. Coleman, Colorectal cancer screening: prospects for molecular stool analysis, Nat Rev Cancer 5 (2005), 199-209.

[24] P.J. Pickhardt, J.R. Choi, I. Hwang, J.A. Butler, M.L. Puckett, H.A. Hildebrandt, R.K. Wong, P.A. Nugent, P.A. Mysliwiec and W.R. Schindler, Computed tomographic virtual colonoscopy to screen for colorectal neoplasia in asymptomatic adults, N Engl J Med 349 (2003), 2191-2200.

[25] D.K. Rex, American College of Gastroenterology action plan for colorectal cancer prevention, Am J Gastroenterol 99 (2004), 574-577.

[26] E. Thiis-Evensen, G.S. Hoff, J. Sauar, F. Langmark, B.M. Majak and M.H. Vatn, Population-based surveillance by colonoscopy: effect on the incidence of colorectal cancer. Telemark Polyp Study I, Scand J Gastroenterol 34 (1999), 414-420.

[27] S.J. Winawer, A.G. Zauber, M.N. Ho, M.J. O'Brien, L.S. Gottlieb, S.S. Sternberg, J.D. Waye, M. Schapiro, J.H. Bond and J.F. Panish, Prevention of colorectal cancer by colonoscopic polypectomy. The National Polyp Study Workgroup, N Engl J Med 329 (1993), 1977-1981.

[28] J.S. Mandel, T.R. Church, J.H. Bond, F. Ederer, M.S. Geisser, S.J. Mongin, D.C. Snover and L.M. Schuman, The effect of fecal occult-blood screening on the incidence of colorectal cancer, $N$ Engl J Med 343 (2000), 1603-1607.

[29] H.M. Fenlon, D.P. Nunes, P.C. Schroy, III, M.A. Barish, P.D. Clarke and J.T. Ferrucci, A comparison of virtual and conventional colonoscopy for the detection of colorectal polyps, N Engl J Med 341 (1999), 1496-1503.

[30] J. Yee, G.A. Akerkar, R.K. Hung, A.M. Steinauer-Gebauer, S.D. Wall and K.R. McQuaid, Colorectal neoplasia: performance characteristics of CT colonography for detection in 300 patients, Radiology 219 (2001), 685-692.

[31] A. Loktionov, I.K. O’Neill, K.R. Silvester, J.H. Cummings, S.J. Middleton and R. Miller, Quantitation of DNA from exfoliated colonocytes isolated from human stool surface as a novel noninvasive screening test for colorectal cancer, Clin Cancer Res 4 (1998), 337-342.

[32] K.A. Boynton, I.C. Summerhayes, D.A. Ahlquist and A.P. Shuber, DNA integrity as a potential marker for stool-based detection of colorectal cancer, Clin Chem 49 (2003), 10581065

[33] D.A. Ahlquist and A.P. Shuber, Stool screening for colorectal cancer: evolution from occult blood to molecular markers, Clin Chim Acta 315 (2002), 157-168.

[34] M. Frattini, D. Balestra, S. Pilotti, L. Bertario and M.A. Pierotti, Tumor location and detection of k-ras mutations in stool from colorectal cancer patients, J Natl Cancer Inst 95 (2003), 72-73.

[35] S. Eguchi, N. Kohara, K. Komuta and T. Kanematsu, Mutations of the p53 gene in the stool of patients with resectable colorectal cancer, Cancer 77 (1996), 1707-1710.

[36] S.M. Powell, N. Zilz, Y. Beazer-Barclay, T.M. Bryan, S.R. Hamilton, S.N. Thibodeau, B. Vogelstein and K.W. Kinzler, APC mutations occur early during colorectal tumorigenesis, Nature 359 (1992), 235-237. 
[37] G. Traverso, A. Shuber, L. Olsson, B. Levin, C. Johnson, S.R. Hamilton, K. Boynton, K.W. Kinzler and B. Vogelstein, Detection of proximal colorectal cancers through analysis of faecal DNA, Lancet 359 (2002), 403-404.

[38] H.M. Muller, M. Oberwalder, H. Fiegl, M. Morandell, G. Goebel, M. Zitt, M. Muhlthaler, D. Ofner, R. Margreiter and M. Widschwendter, Methylation changes in faecal DNA: a marker for colorectal cancer screening? Lancet 363 (2004), 1283-1285.

[39] A.M. Abulafi and N.S. Williams, Local recurrence of colorectal cancer: the problem, mechanisms, management and adjuvant therapy, Br J Surg 81 (1994), 7-19.

[40] M. Rosen, L. Chan, R.W. Beart, Jr., P. Vukasin and G. Anthone, Follow-up of colorectal cancer: a meta-analysis, Dis Colon Rectum 41 (1998), 1116-1126.

[41] Y. Fong and L.H. Blumgart, Hepatic colorectal metastasis: current status of surgical therapy, Oncology (Williston Park) 12 (1998), 1489-1498.

[42] M. Rees, G. Plant and S. Bygrave, Late results justify resection for multiple hepatic metastases from colorectal cancer, Br J Surg 84 (1997), 1136-1140.

[43] H. Ike, H. Shimada, S. Togo, S. Yamaguchi, Y. Ichikawa and K. Tanaka, Sequential resection of lung metastasis following partial hepatectomy for colorectal cancer, Br J Surg 89 (2002), 1164-1168.

[44] M. Zitt, G. Muhlmann, H. Weiss, R. Kafka-Ritsch, M. Oberwalder, W. Kirchmayr, R. Margreiter, D. Ofner and A. Klaus, Assessment of risk-independent follow-up to detect asymptomatic recurrence after curative resection of colorectal cancer, Langenbecks Arch Surg (2006).

[45] C.E. Desch, A.B. Benson, III, M.R. Somerfield, P.J. Flynn, C. Krause, C.L. Loprinzi, B.D. Minsky, D.G. Pfister, K.S. Virgo and N.J. Petrelli, Colorectal cancer surveillance: 2005 update of an American Society of Clinical Oncology practice guideline, J Clin Oncol 23 (2005), 8512-8519.

[46] A.G. Renehan, M. Egger, M.P. Saunders and S.T. O'Dwyer, Impact on survival of intensive follow up after curative resection for colorectal cancer: systematic review and metaanalysis of randomised trials, BMJ 324 (2002), 813.

[47] H.M. Muller and M. Widschwendter, Methylated DNA as a possible screening marker for neoplastic disease in several body fluids, Expert Rev Mol Diagn 3 (2003), 443-458.

[48] A. Widschwendter, H.M. Muller, H. Fiegl, L. Ivarsson, A. Wiedemair, E. Muller-Holzner, G. Goebel, C. Marth and M. Widschwendter, DNA methylation in serum and tumors of cervical cancer patients, Clin Cancer Res 10 (2004), 565571.

[49] A. Widschwendter, L. Ivarsson, A. Blassnig, H.M. Muller, H. Fiegl, A. Wiedemair, E. Muller-Holzner, G. Goebel, C. Marth and $\mathrm{M}$. Widschwendter, $\mathrm{CDH} 1$ and $\mathrm{CDH} 13$ methylation in serum is an independent prognostic marker in cervical cancer patients, Int J Cancer 109 (2004), 163-166.

[50] H.M. Muller, A. Widschwendter, H. Fiegl, L. Ivarsson, G. Goebel, E. Perkmann, C. Marth and M. Widschwendter, DNA methylation in serum of breast cancer patients: an independent prognostic marker, Cancer Res 63 (2003), 76417645 .

[51] H.M. Muller, L. Ivarsson, H. Schrocksnadel, H. Fiegl, A. Widschwendter, G. Goebel, S. Kilga-Nogler, H. Philadelphy, W. Gutter, C. Marth and M. Widschwendter, DNA methylation changes in sera of women in early pregnancy are similar to those in advanced breast cancer patients, Clin Chem $\mathbf{5 0}$ (2004), 1065-1068.
[52] H.M. Muller, H. Fiegl, A. Widschwendter and M. Widschwendter, Prognostic DNA Methylation Marker in Serum of Cancer Patients, Ann N Y Acad Sci 1022 (2004), 44-49.

[53] C. Lengauer, K.W. Kinzler and B. Vogelstein, Genetic instabilities in human cancers, Nature 396 (1998), 643-649.

[54] K.W. Kinzler and B. Vogelstein, Lessons from hereditary colorectal cancer, Cell 87 (1996), 159-170.

[55] W.M. Grady, Epigenetic events in the colorectum and in colon cancer, Biochem Soc Trans 33 (2005), 684-688.

[56] B.C. Morson, Evolution of cancer of the colon and rectum, Cancer 34 (1974), suppl-9.

[57] J.R. Jass, V.L. Whitehall, J. Young and B.A. Leggett, Emerging concepts in colorectal neoplasia, Gastroenterology 123 (2002), 862-876.

[58] T. Kambara, L.A. Simms, V.L. Whitehall, K.J. Spring, C.V. Wynter, M.D. Walsh, M.A. Barker, S. Arnold, A. McGivern, N. Matsubara, N. Tanaka, T. Higuchi, J. Young, J.R. Jass and B.A. Leggett, BRAF mutation is associated with DNA methylation in serrated polyps and cancers of the colorectum, Gut 53 (2004), 1137-1144.

[59] C.V. Wynter, M.D. Walsh, T. Higuchi, B.A. Leggett, J. Young and J.R. Jass, Methylation patterns define two types of hyperplastic polyp associated with colorectal cancer, Gut $\mathbf{5 3}$ (2004), 573-580.

[60] S. Kozuka, M. Nogaki, T. Ozeki and S. Masumori, Premalignancy of the mucosal polyp in the large intestine: II. Estimation of the periods required for malignant transformation of mucosal polyps, Dis Colon Rectum 18 (1975), 494-500.

[61] M.J. O’Brien, S.J. Winawer, A.G. Zauber, L.S. Gottlieb, S.S. Sternberg, B. Diaz, G.R. Dickersin, S. Ewing, S. Geller and D. Kasimian, The National Polyp Study. Patient and polyp characteristics associated with high-grade dysplasia in colorectal adenomas, Gastroenterology 98 (1990), 371-379.

[62] J.M. Rhodes, Colorectal cancer screening in the UK: Joint Position Statement by the British Society of Gastroenterology, The Royal College of Physicians, and The Association of Coloproctology of Great Britain and Ireland, Gut $\mathbf{4 6}$ (2000), 746-748

[63] S. Kudo, H. Kashida and T. Tamura, Early colorectal cancer: flat or depressed type, J Gastroenterol Hepatol 15 Suppl (2000), D66-D70.

[64] N. Umetani, S. Sasaki, T. Masaki, T. Watanabe, K. Matsuda and T. Muto, Involvement of APC and K-ras mutation in nonpolypoid colorectal tumorigenesis, Br J Cancer 82 (2000), 9-15.

[65] S. Hirota, S. Kudo, S. Hosobe, T. Kobayashi, M. Himori, M. Ikeda, Y. Takemoto, M. Nomoto, Y. Aoyagi and H. Asakura, p53 immunoreactive stain and early colorectal adenocarcinomas, Eur J Cancer 31A (1995), 2220-2222.

[66] R. Wada, S. Matsukuma, H. Abe, N. Kuwabara, K. Suda, A. Arakawa and S. Kitamura, Histopathological studies of superficial-type early colorectal carcinoma, Cancer $\mathbf{7 7}$ (1996), 44-50.

[67] D.C. Chung and A.K. Rustgi, DNA mismatch repair and cancer, Gastroenterology 109 (1995), 1685-1699.

[68] S.N. Thibodeau, G. Bren and D. Schaid, Microsatellite instability in cancer of the proximal colon, Science 260 (1993), 816-819.

[69] I.M. Frayling, Microsatellite instability, Gut 45 (1999), 1-4.

[70] R.H. Giles, J.H. van Es and H. Clevers, Caught up in a Wnt storm: Wnt signaling in cancer, Biochim Biophys Acta 1653 (2003), 1-24.

[71] J. Groden, A. Thliveris, W. Samowitz, M. Carlson, L. Gelbert, H. Albertsen, G. Joslyn, J. Stevens, L. Spirio and M. 
Robertson, Identification and characterization of the familial adenomatous polyposis coli gene, Cell 66 (1991), 589-600.

[72] K.W. Kinzler, M.C. Nilbert, L.K. Su, B. Vogelstein, T.M. Bryan, D.B. Levy, K.J. Smith, A.C. Preisinger, P. Hedge and D. McKechnie, Identification of FAP locus genes from chromosome 5q21, Science 253 (1991), 661-665.

[73] H. Nagase and Y. Nakamura, Mutations of the APC (adenomatous polyposis coli) gene, Hum Mutat 2 (1993), 425-434

[74] P.J. Morin, A.B. Sparks, V. Korinek, N. Barker, H. Clevers, B. Vogelstein and K.W. Kinzler, Activation of beta-cateninTcf signaling in colon cancer by mutations in beta-catenin or APC, Science 275 (1997), 1787-1790.

[75] W. Liu, X. Dong, M. Mai, R.S. Seelan, K. Taniguchi, K.K. Krishnadath, K.C. Halling, J.M. Cunningham, L.A. Boardman, C. Qian, E. Christensen, S.S. Schmidt, P.C. Roche, D.I. Smith and S.N. Thibodeau, Mutations in AXIN2 cause colorectal cancer with defective mismatch repair by activating beta-catenin/TCF signalling, Nat Genet 26 (2000), 146-147.

[76] S.M. Uthoff, M.R. Eichenberger, T.L. McAuliffe, C.J. Hamilton and S. Galandiuk, Wingless-type frizzled protein receptor signaling and its putative role in human colon cancer, $\mathrm{Mol}$ Carcinog 31 (2001), 56-62.

[77] M. Widschwendter and P.A. Jones, DNA methylation and breast carcinogenesis, Oncogene 21 (2002), 5462-5482.

[78] P.A. Jones and S.B. Baylin, The fundamental role of epigenetic events in cancer, Nat Rev Genet 3 (2002), 415-428.

[79] P.W. Laird, Early detection: The power and the promise of DNA methylation markers, Nat Rev Cancer 3 (2003), 253266.

[80] P.A. Jones and P.W. Laird, Cancer epigenetics comes of age, Nat Genet 21 (1999), 163-167.

[81] A. Bird, DNA methylation patterns and epigenetic memory, Genes Dev 16 (2002), 6-21.

[82] H. Fiegl, C. Gattringer, A. Widschwendter, A. Schneitter, A. Ramoni, D. Sarlay, I. Gaugg, G. Goebel, H.M. Muller, E. Mueller-Holzner, C. Marth and M. Widschwendter, Methylated DNA collected by tampons - a new tool to detect endometrial cancer, Cancer Epidemiol Biomarkers Prev 13 (2004), 882-888.

[83] H.M. Muller, S. Millinger, H. Fiegl, G. Goebel, L. Ivarsson, A. Widschwendter, E. Muller-Holzner, C. Marth and M. Widschwendter, Analysis of methylated genes in peritoneal fluids of ovarian cancer patients: a new prognostic tool, Clin Chem 50 (2004), 2171-2173.

[84] A.M. Jubb, S.M. Bell and P. Quirke, Methylation and colorectal cancer, J Pathol 195 (2001), 111-134.

[85] J.P. Issa, N. Ahuja, M. Toyota, M.P. Bronner and T.A. Brentnall, Accelerated age-related cpg island methylation in ulcerative colitis, Cancer Res 61 (2001), 3573-3577.

[86] F. Sato, N. Harpaz, D. Shibata, Y. Xu, J. Yin, Y. Mori, T.T. Zou, S. Wang, K. Desai, A. Leytin, F.M. Selaru, J.M. Abraham and S.J. Meltzer, Hypermethylation of the p14(ARF) gene in ulcerative colitis-associated colorectal carcinogenesis, Cancer Res 62 (2002), 1148-1151.

[87] F. Sato, D. Shibata, N. Harpaz, Y. Xu, J. Yin, Y. Mori, S. Wang, A. Olaru, E. Deacu, F.M. Selaru, M.C. Kimos, P. Hytiroglou, J. Young, B. Leggett, A.F. Gazdar, S. Toyooka, J.M. Abraham and S.J. Meltzer, Aberrant methylation of the HPP1 gene in ulcerative colitis-associated colorectal carcinoma, Cancer Res 62 (2002), 6820-6822.

[88] J.G. Herman, A. Umar, K. Polyak, J.R. Graff, N. Ahuja, J.P. Issa, S. Markowitz, J.K. Willson, S.R. Hamilton, K.W. Kinzler, M.F. Kane, R.D. Kolodner, B. Vogelstein, T.A. Kunkel and S.B. Baylin, Incidence and functional consequences of
hMLH1 promoter hypermethylation in colorectal carcinoma, Proc Natl Acad Sci USA 95 (1998), 6870-6875.

[89] M.F. Kane, M. Loda, G.M. Gaida, J. Lipman, R. Mishra, H. Goldman, J.M. Jessup and R. Kolodner, Methylation of the hMLH1 promoter correlates with lack of expression of hMLH1 in sporadic colon tumors and mismatch repairdefective human tumor cell lines, Cancer Res 57 (1997), 808-811.

[90] M.L. Veigl, L. Kasturi, J. Olechnowicz, A.H. Ma, J.D. Lutterbaugh, S. Periyasamy, G.M. Li, J. Drummond, P.L. Modrich, W.D. Sedwick and S.D. Markowitz, Biallelic inactivation of hMLH1 by epigenetic gene silencing, a novel mechanism causing human MSI cancers, Proc Natl Acad Sci USA 95 (1998), 8698-8702.

[91] G. Deng, A. Chen, J. Hong, H.S. Chae and Y.S. Kim, Methylation of $\mathrm{CpG}$ in a small region of the hMLH1 promoter invariably correlates with the absence of gene expression, Cancer Res 59 (1999), 2029-2033.

[92] N.J. Hawkins and R.L. Ward, Sporadic colorectal cancers with microsatellite instability and their possible origin in hyperplastic polyps and serrated adenomas, $J$ Natl Cancer Inst 93 (2001), 1307-1313.

[93] H. Nakagawa, G.J. Nuovo, E.E. Zervos, E.W. Martin, Jr., R. Salovaara, L.A. Aaltonen and C.A. de la, Age-related hypermethylation of the 5' region of MLH1 in normal colonic mucosa is associated with microsatellite-unstable colorectal cancer development, Cancer Res 61 (2001), 6991-6995.

[94] S.B. Baylin and J.G. Herman, DNA hypermethylation in tumorigenesis: epigenetics joins genetics, Trends Genet 16 (2000), 168-174.

[95] M. Toyota, C. Ho, N. Ahuja, K.W. Jair, Q. Li, M. OheToyota, S.B. Baylin and J.P. Issa, Identification of differentially methylated sequences in colorectal cancer by methylated CpG island amplification, Cancer Res 59 (1999), 23072312.

[96] Z. Petko, M. Ghiassi, A. Shuber, J. Gorham, W. Smalley, M.K. Washington, S. Schultenover, S. Gautam, S.D. Markowitz and W.M. Grady, Aberrantly methylated CDKN2A, MGMT, and MLH1 in colon polyps and in fecal DNA from patients with colorectal polyps, Clin Cancer Res 11 (2005), 1203-1209.

[97] A. Rashid, L. Shen, J.S. Morris, J.P. Issa and S.R. Hamilton, $\mathrm{CpG}$ island methylation in colorectal adenomas, Am J Pathol 159 (2001), 1129-1135.

[98] N. Ahuja, Q. Li, A.L. Mohan, S.B. Baylin and J.P. Issa, Aging and DNA methylation in colorectal mucosa and cancer, Cancer Res 58 (1998), 5489-5494.

[99] M. Esteller, Epigenetic lesions causing genetic lesions in human cancer: promoter hypermethylation of DNA repair genes, Eur J Cancer 36 (2000), 2294-2300.

[100] A.O. Chan, R.R. Broaddus, P.S. Houlihan, J.P. Issa, S.R. Hamilton and A. Rashid, $\mathrm{CpG}$ island methylation in aberrant crypt foci of the colorectum, Am J Pathol 160 (2002), 18231830.

[101] H. Li, L. Myeroff, D. Smiraglia, M.F. Romero, T.P. Pretlow, L. Kasturi, J. Lutterbaugh, R.M. Rerko, G. Casey, J.P. Issa, J. Willis, J.K. Willson, C. Plass and S.D. Markowitz, SLC5A8, a sodium transporter, is a tumor suppressor gene silenced by methylation in human colon aberrant crypt foci and cancers, Proc Natl Acad Sci USA 100 (2003), 8412-8417.

[102] M. Toyota, N. Ahuja, M. Ohe-Toyota, J.G. Herman, S.B. Baylin and J.P. Issa, CpG island methylator phenotype in colorectal cancer, Proc Natl Acad Sci USA 96 (1999), 86818686 
[103] A.H. Bai, J.H. Tong, K.F. To, M.W. Chan, E.P. Man, K.W. Lo, J.F. Lee, J.J. Sung and W.K. Leung, Promoter hypermethylation of tumor-related genes in the progression of colorectal neoplasia, Int J Cancer 112 (2004), 846-853.

[104] S. Lee, K.S. Hwang, H.J. Lee, J.S. Kim and G.H. Kang, Aberrant $\mathrm{CpG}$ island hypermethylation of multiple genes in colorectal neoplasia, Lab Invest 84 (2004), 884-893.

[105] G.E. Lind, L. Thorstensen, T. Lovig, G.I. Meling, R. Hamelin, T.O. Rognum, M. Esteller and R.A. Lothe, A CpG island hypermethylation profile of primary colorectal carcinomas and colon cancer cell lines, Mol Cancer 3 (2004), 28.

[106] K. Koinuma, Y. Yamashita, W. Liu, H. Hatanaka, K. Kurashina, T. Wada, S. Takada, R. Kaneda, Y.L. Choi, S.I. Fujiwara, Y. Miyakura, H. Nagai and H. Mano, Epigenetic silencing of AXIN2 in colorectal carcinoma with microsatellite instability, Oncogene 25 (2006), 139-146.

[107] G.M. Caldwell, C. Jones, K. Gensberg, S. Jan, R.G. Hardy, P. Byrd, S. Chughtai, Y. Wallis, G.M. Matthews and D.G. Morton, The Wnt antagonist sFRP1 in colorectal tumorigenesis, Cancer Res 64 (2004), 883-888.

[108] H. Suzuki, E. Gabrielson, W. Chen, R. Anbazhagan, M. van Engeland, M.P. Weijenberg, J.G. Herman and S.B. Baylin, A genomic screen for genes upregulated by demethylation and histone deacetylase inhibition in human colorectal cancer, Nat Genet 31 (2002), 141-149.

[109] B. He, N. Reguart, L. You, J. Mazieres, Z. Xu, A.Y. Lee, I. Mikami, F. McCormick and D.M. Jablons, Blockade of Wnt1 signaling induces apoptosis in human colorectal cancer cells containing downstream mutations, Oncogene 24 (2005), 3054-3058.

[110] O. Aguilera, M.F. Fraga, E. Ballestar, M.F. Paz, M. Herranz, J. Espada, J.M. Garcia, A. Munoz, M. Esteller and J.M. Gonzalez-Sancho, Epigenetic inactivation of the Wnt antagonist DICKKOPF-1 (DKK-1) gene in human colorectal cancer, Oncogene (2006).

[111] B. Shapiro, M. Chakrabarty, E.M. Cohn and S.A. Leon, Determination of circulating DNA levels in patients with benign or malignant gastrointestinal disease, Cancer 51 (1983), 2116-2120.

[112] W.M. Grady, A. Rajput, J.D. Lutterbaugh and S.D. Markowitz, Detection of aberrantly methylated hMLH1 promoter DNA in the serum of patients with microsatellite unstable colon cancer, Cancer Res 61 (2001), 900-902.

[113] H. Nakayama, K. Hibi, M. Taguchi, T. Takase, T. Yamazaki, Y. Kasai, K. Ito, S. Akiyama and A. Nakao, Molecular detection of p16 promoter methylation in the serum of colorectal cancer patients, Cancer Lett 188 (2002), 115-119.

[114] H.Z. Zou, B.M. Yu, Z.W. Wang, J.Y. Sun, H. Cang, F. Gao, D.H. Li, R. Zhao, G.G. Feng and J. Yi, Detection of aberrant p16 methylation in the serum of colorectal cancer patients, Clin Cancer Res 8 (2002), 188-191.

[115] T. Lecomte, A. Berger, F. Zinzindohoue, S. Micard, B. Landi, H. Blons, P. Beaune, P.H. Cugnenc and P. Laurent-Puig, Detection of free-circulating tumor-associated DNA in plasma of colorectal cancer patients and its association with prognosis, Int J Cancer 100 (2002), 542-548.

[116] Y. Sakamoto, R. Kitazawa, S. Maeda and S. Kitazawa, Methylation of CpG loci in 5'-flanking region alters steadystate expression of adenomatous polyposis coli gene in colon cancer cell lines, J Cell Biochem 80 (2001), 415-423.

[117] M. Murai, M. Toyota, H. Suzuki, A. Satoh, Y. Sasaki, K. Akino, M. Ueno, F. Takahashi, M. Kusano, H. Mita, K Yanagihara, T. Endo, Y. Hinoda, T. Tokino and K. Imai,
Aberrant methylation and silencing of the BNIP3 gene in colorectal and gastric cancer, Clin Cancer Res 11 (2005), 1021-1027.

[118] S. Toyooka, K.O. Toyooka, K. Harada, K. Miyajima, P. Makarla, U.G. Sathyanarayana, J. Yin, F. Sato, N. Shivapurkar, S.J. Meltzer and A.F. Gazdar, Aberrant methylation of the CDH13 (H-cadherin) promoter region in colorectal cancers and adenomas, Cancer Res 62 (2002), 3382-3386.

[119] E. Miotto, S. Sabbioni, A. Veronese, G.A. Calin, S. Gullini, A. Liboni, L. Gramantieri, L. Bolondi, E. Ferrazzi, R. Gafa, G. Lanza and M. Negrini, Frequent aberrant methylation of the $\mathrm{CDH} 4$ gene promoter in human colorectal and gastric cancer, Cancer Res 64 (2004), 8156-8159.

[120] S. Zheng, P. Chen, A. McMillan, A. Lafuente, M.J. Lafuente, A. Ballesta, M. Trias and J.K. Wiencke, Correlations of partial and extensive methylation at the p14(ARF) locus with reduced mRNA expression in colorectal cancer cell lines and clinicopathological features in primary tumors, Carcinogenesis 21 (2000), 2057-2064.

[121] K.J. Wagner, W.N. Cooper, R.G. Grundy, G. Caldwell, C. Jones, R.B. Wadey, D. Morton, P.N. Schofield, W. Reik, F. Latif and E.R. Maher, Frequent RASSF1A tumour suppressor gene promoter methylation in Wilms' tumour and colorectal cancer, Oncogene 21 (2002), 7277-7282.

[122] N.A. Wong, M.P. Britton, G.S. Choi, T.K. Stanton, D.C. Bicknell, J.L. Wilding and W.F. Bodmer, Loss of CDX1 expression in colorectal carcinoma: promoter methylation, mutation, and loss of heterozygosity analyses of 37 cell lines, Proc Natl Acad Sci USA 101 (2004), 574-579.

[123] E.R. Suh, C.S. Ha, E.B. Rankin, M. Toyota and P.G. Traber, DNA methylation down-regulates CDX1 gene expression in colorectal cancer cell lines, J Biol Chem 277 (2002), 3579535800 .

[124] P.G. Corn, M.K. Summers, F. Fogt, A.K. Virmani, A.F. Gazdar, T.D. Halazonetis and W.S. El Deiry, Frequent hypermethylation of the 5' $\mathrm{CpG}$ island of the mitotic stress checkpoint gene Chfr in colorectal and non-small cell lung cancer, Carcinogenesis 24 (2003), 47-51.

[125] P.K. Sengupta, E.M. Smith, K. Kim, M.J. Murnane and B.D. Smith, DNA hypermethylation near the transcription start site of collagen alpha2(I) gene occurs in both cancer cell lines and primary colorectal cancers, Cancer Res 63 (2003), 1789-1797.

[126] A. Satoh, M. Toyota, F. Itoh, T. Kikuchi, T. Obata, Y. Sasaki, H. Suzuki, A. Yawata, M. Kusano, M. Fujita, M. Hosokawa, K. Yanagihara, T. Tokino and K. Imai, DNA methylation and histone deacetylation associated with silencing DAP kinase gene expression in colorectal and gastric cancers, Br J Cancer 86 (2002), 1817-1823.

[127] J. Wang, H. Kataoka, M. Suzuki, N. Sato, R. Nakamura, H. Tao, K. Maruyama, J. Isogaki, S. Kanaoka, M. Ihara, M. Tanaka, M. Kanamori, T. Nakamura, K. Shinmura and H. Sugimura, Downregulation of EphA7 by hypermethylation in colorectal cancer, Oncogene 24 (2005), 5637-5647.

[128] Y. Akiyama, N. Watkins, H. Suzuki, K.W. Jair, M. van Engeland, M. Esteller, H. Sakai, C.Y. Ren, Y. Yuasa, J.G. Herman and S.B. Baylin, GATA-4 and GATA-5 transcription factor genes and potential downstream antitumor target genes are epigenetically silenced in colorectal and gastric cancer, $\mathrm{Mol}$ Cell Biol 23 (2003), 8429-8439.

[129] N. Umetani, H. Takeuchi, A. Fujimoto, M. Shinozaki, A.J. Bilchik and D.S. Hoon, Epigenetic inactivation of ID4 in colorectal carcinomas correlates with poor differentiation and 
unfavorable prognosis, Clin Cancer Res 10 (2004), 74757483 .

[130] J. Frigola, M. Munoz, S.J. Clark, V. Moreno, G. Capella and M.A. Peinado, Hypermethylation of the prostacyclin synthase (PTGIS) promoter is a frequent event in colorectal cancer and associated with aneuploidy, Oncogene 24 (2005), $7320-7326$.

[131] M. Toyota, L. Shen, M. Ohe-Toyota, S.R. Hamilton, F.A. Sinicrope and J.P. Issa, Aberrant methylation of the Cyclooxygenase $2 \mathrm{CpG}$ island in colorectal tumors, Cancer Res 60 (2000), 4044-4048.

[132] K. Akino, M. Toyota, H. Suzuki, H. Mita, Y. Sasaki, M. OheToyota, J.P. Issa, Y. Hinoda, K. Imai and T. Tokino, The Ras effector RASSF2 is a novel tumor-suppressor gene in human colorectal cancer, Gastroenterology 129 (2005), 156-169.

[133] J.L. Ku, S.B. Kang, Y.K. Shin, H.C. Kang, S.H. Hong, I.J. Kim, J.H. Shin, I.O. Han and J.G. Park, Promoter hypermethylation downregulates RUNX3 gene expression in colorectal cancer cell lines, Oncogene 23 (2004), 6736-6742.

[134] H.R. Moinova, W.D. Chen, L. Shen, D. Smiraglia, J. Olechnowicz, L. Ravi, L. Kasturi, L. Myeroff, C. Plass, R. Parsons, J. Minna, J.K. Willson, S.B. Green, J.P. Issa and S.D. Markowitz, HLTF gene silencing in human colon cancer, Proc Natl Acad Sci USA 99 (2002), 4562-4567.

[135] M.P. Ebert, S.H. Mooney, L. Tonnes-Priddy, J. Lograsso, J. Hoffmann, J. Chen, C. Rocken, H.U. Schulz, P. Malfertheiner and C. Lofton-Day, Hypermethylation of the TPEF/HPP1 gene in primary and metastatic colorectal cancers, Neoplasia 7 (2005), 771-778

[136] J. Young, K.G. Biden, L.A. Simms, P. Huggard, R. Karamatic, H.J. Eyre, G.R. Sutherland, N. Herath, M. Barker, G.J. Anderson, D.R. Fitzpatrick, G.A. Ramm, J.R. Jass and B.A. Leggett, HPP1: a transmembrane protein-encoding gene commonly methylated in colorectal polyps and cancers, Proc Natl Acad Sci USA 98 (2001), 265-270.

[137] M. Van Rijnsoever, F. Grieu, H. Elsaleh, D. Joseph and B. Iacopetta, Characterisation of colorectal cancers showing hypermethylation at multiple CpG islands, Gut 51 (2002), 797802.

[138] S.J. Park, A. Rashid, J.H. Lee, S.G. Kim, S.R. Hamilton and T.T. Wu, Frequent CpG island methylation in serrated adenomas of the colorectum, Am J Pathol 162 (2003), 815822.

[139] J.C. Kim, K.H. Koo, S.A. Roh, Y.K. Cho, H.C. Kim, C.S. Yu, H.J. Kim, J.S. Kim and M.K. Cho, Genetic and epigenetic changes in the APC gene in sporadic colorectal carcinoma with synchronous adenoma, Int J Colorectal Dis 18 (2003), 203-209.

[140] Z. Xiong, A.H. Wu, C.M. Bender, J.L. Tsao, C. Blake, D. Shibata, P.A. Jones, M.C. Yu, R.K. Ross and P.W. Laird, Mismatch repair deficiency and $\mathrm{CpG}$ island hypermethylation in sporadic colon adenocarcinomas, Cancer Epidemiol Biomarkers Prev 10 (2001), 799-803.

[141] M. Esteller, A. Sparks, M. Toyota, M. Sanchez-Cespedes, G. Capella, M.A. Peinado, S. Gonzalez, G. Tarafa, D. Sidransky, S.J. Meltzer, S.B. Baylin and J.G. Herman, Analysis of adenomatous polyposis coli promoter hypermethylation in human cancer, Cancer Res 60 (2000), 4366-4371.

[142] T. Kanazawa, T. Watanabe, S. Kazama, T. Tada, S. Koketsu and H. Nagawa, Poorly differentiated adenocarcinoma and mucinous carcinoma of the colon and rectum show higher rates of loss of heterozygosity and loss of E-cadherin expression due to methylation of promoter region, Int J Cancer 102 (2002), 225-229.
[143] G.A. Garinis, P.G. Menounos, N.E. Spanakis, K. Papadopoulos, G. Karavitis, I. Parassi, E. Christeli, G.P. Patrinos, E.N. Manolis and G. Peros, Hypermethylation-associated transcriptional silencing of E-cadherin in primary sporadic colorectal carcinomas, J Pathol 198 (2002), 442-449.

[144] K. Hibi, Y. Kodera, K. Ito, S. Akiyama and A. Nakao, Aberrant methylation of HLTF, SOCS-1, and CDH13 genes is shown in colorectal cancers without lymph node metastasis, Dis Colon Rectum 48 (2005), 1282-1286.

[145] K. Hibi, H. Nakayama, Y. Kodera, K. Ito, S. Akiyama and A. Nakao, CDH13 promoter region is specifically methylated in poorly differentiated colorectal cancer, $\mathrm{Br} J$ Cancer 90 (2004), 1030-1033.

[146] J. Yi, Z.W. Wang, H. Cang, Y.Y. Chen, R. Zhao, B.M. Yu and X.M. Tang, p16 gene methylation in colorectal cancers associated with Duke's staging, World J Gastroenterol 7 (2001), $722-725$.

[147] M. Toyota, M. Ohe-Toyota, N. Ahuja and J.P. Issa, Distinct genetic profiles in colorectal tumors with or without the $\mathrm{CpG}$ island methylator phenotype, Proc Natl Acad Sci USA 97 (2000), 710-715.

[148] H. Nakayama, K. Hibi, T. Takase, T. Yamazaki, Y. Kasai, K. Ito, S. Akiyama and A. Nakao, Molecular detection of p16 promoter methylation in the serum of recurrent colorectal cancer patients, Int J Cancer 105 (2003), 491-493.

[149] C.V. Wynter, T. Kambara, M.D. Walsh, B.A. Leggett, J. Young and J.R. Jass, DNA methylation patterns in adenomas from FAP, multiple adenoma and sporadic colorectal carcinoma patients, Int J Cancer 118 (2006), 907-915.

[150] S. Yamaguchi, T. Asao, J. Nakamura, M. Ide and H. Kuwano, High frequency of DAP-kinase gene promoter methylation in colorectal cancer specimens and its identification in serum, Cancer Lett 194 (2003), 99-105.

[151] K. Tominaga, S. Fujii, K. Mukawa, M. Fujita, K. Ichikawa, S. Tomita, Y. Imai, K. Kanke, Y. Ono, A. Terano, H. Hiraishi and T. Fujimori, Prediction of colorectal neoplasia by quantitative methylation analysis of estrogen receptor gene in nonneoplastic epithelium from patients with ulcerative colitis, Clin Cancer Res 11 (2005), 8880-8885.

[152] I. Gazzoli, M. Loda, J. Garber, S. Syngal and R.D. Kolodner, A hereditary nonpolyposis colorectal carcinoma case associated with hypermethylation of the MLH1 gene in normal tissue and loss of heterozygosity of the unmethylated allele in the resulting microsatellite instability-high tumor, Cancer Res 62 (2002), 3925-3928.

[153] A.S. Fleisher, M. Esteller, N. Harpaz, A. Leytin, A. Rashid, Y. Xu, J. Liang, O.C. Stine, J. Yin, T.T. Zou, J.M. Abraham, D. Kong, K.T. Wilson, S.P. James, J.G. Herman and S.J. Meltzer, Microsatellite instability in inflammatory bowel disease-associated neoplastic lesions is associated with hypermethylation and diminished expression of the DNA mismatch repair gene, hMLH1, Cancer Res 60 (2000), 48644868.

[154] B. Shannon, P. Kay, A. House and B. Iacopetta, Hypermethylation of the MYF-3 gene in colorectal cancers: associations with pathological features and with microsatellite instability, Int J Cancer 84 (1999), 109-113.

[155] M. van Engeland, G.M. Roemen, M. Brink, M.M. Pachen, M.P. Weijenberg, A.P. de Bruine, J.W. Arends, P.A. van den Brandt, A.F. de Goeij and J.G. Herman, K-ras mutations and RASSF1A promoter methylation in colorectal cancer, Oncogene 21 (2002), 3792-3795.

[156] C. Oliveira, S. Velho, E. Domingo, A. Preto, R.M. Hofstra, R. Hamelin, H. Yamamoto, R. Seruca and S. Schwartz Jr, Con- 
comitant RASSF1A hypermethylation and KRAS/BRAF mutations occur preferentially in MSI sporadic colorectal cancer, Oncogene 24 (2005), 7630-7634.

[157] Y. Imamura, K. Hibi, M. Koike, M. Fujiwara, Y. Kodera, K. Ito and A. Nakao, RUNX3 promoter region is specifically methylated in poorly-differentiated colorectal cancer, Anticancer Res 25 (2005), 2627-2630.

[158] W.D. Chen, Z.J. Han, J. Skoletsky, J. Olson, J. Sah, L. Myeroff, P. Platzer, S. Lu, D. Dawson, J. Willis, T.P. Pretlow, J. Lutterbaugh, L. Kasturi, J.K. Willson, J.S. Rao, A. Shuber and S.D. Markowitz, Detection in fecal DNA of colon cancerspecific methylation of the nonexpressed vimentin gene, $J$ Natl Cancer Inst 97 (2005), 1124-1132.
[159] K. Lenhard, G.T. Bommer, S. Asutay, R. Schauer, T. Brabletz, B. Goke, R. Lamerz and F.T. Kolligs, Analysis of promoter methylation in stool: a novel method for the detection of colorectal cancer, Clin Gastroenterol Hepatol 3 (2005), 142-149.

[160] W.K. Leung, K.F. To, E.P. Man, M.W. Chan, A.H. Bai, A.J. Hui, F.K. Chan and J.J. Sung, Quantitative detection of promoter hypermethylation in multiple genes in the serum of patients with colorectal cancer, Am J Gastroenterol 100 (2005), 2274-2279. 


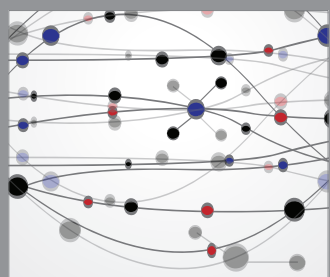

The Scientific World Journal
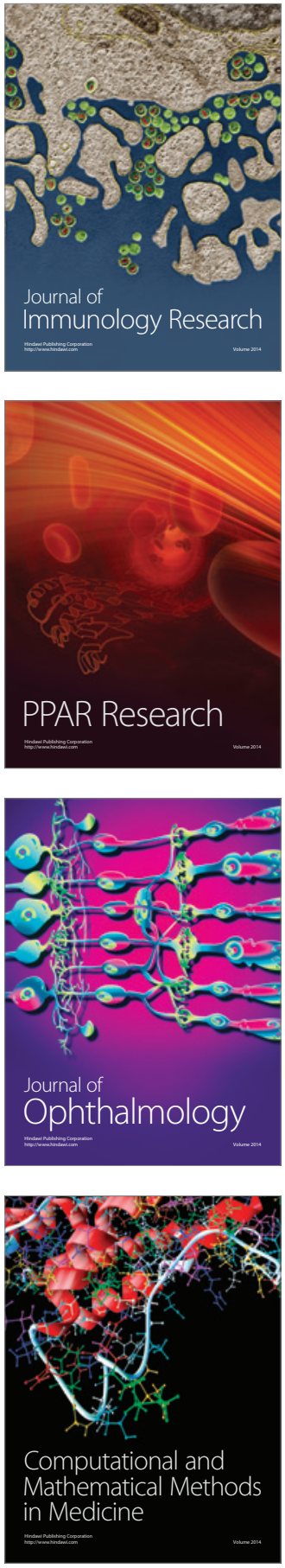

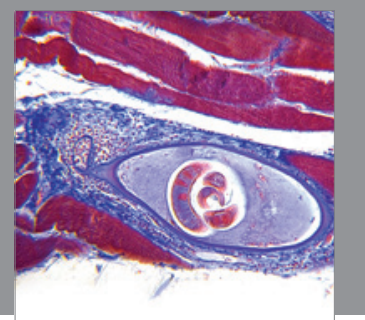

Gastroenterology

Research and Practice
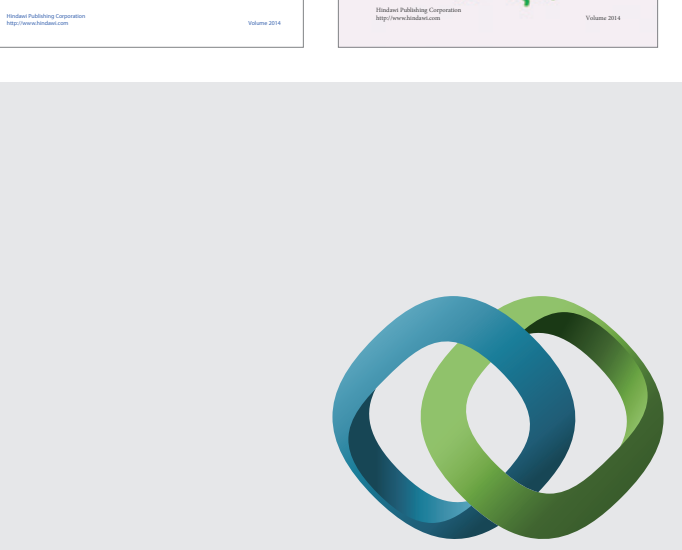

\section{Hindawi}

Submit your manuscripts at

http://www.hindawi.com
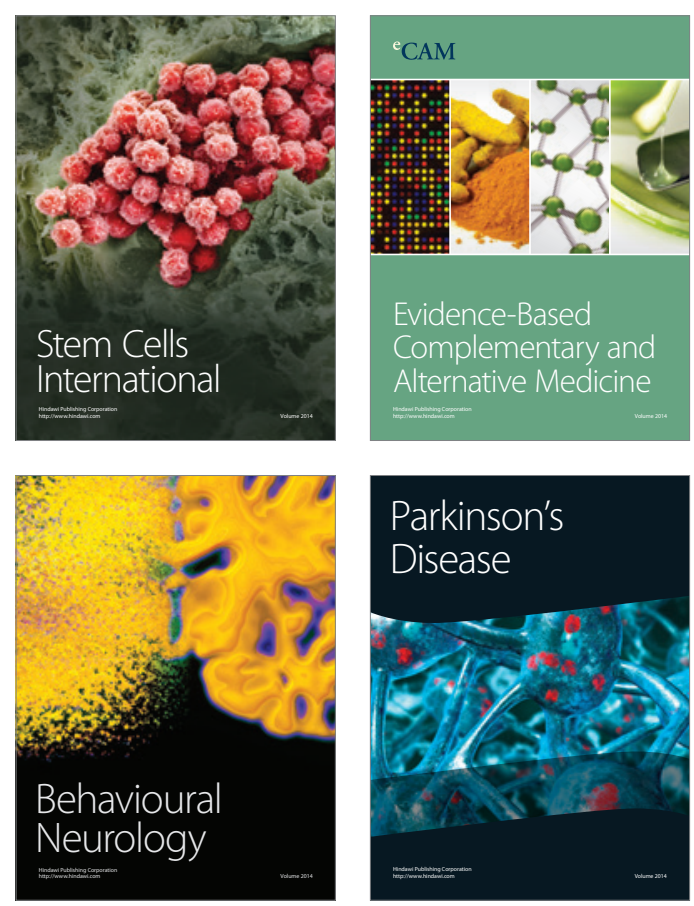

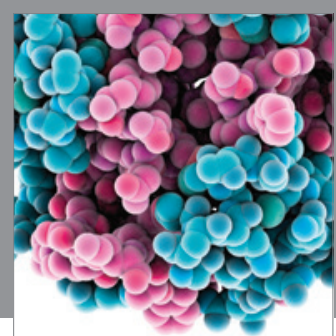

Journal of
Diabetes Research

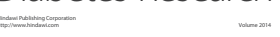

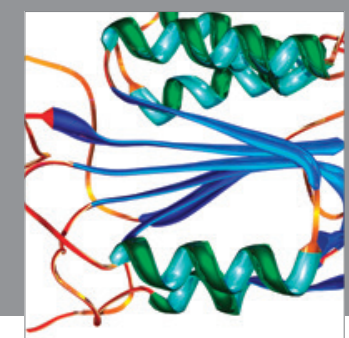

Disease Markers
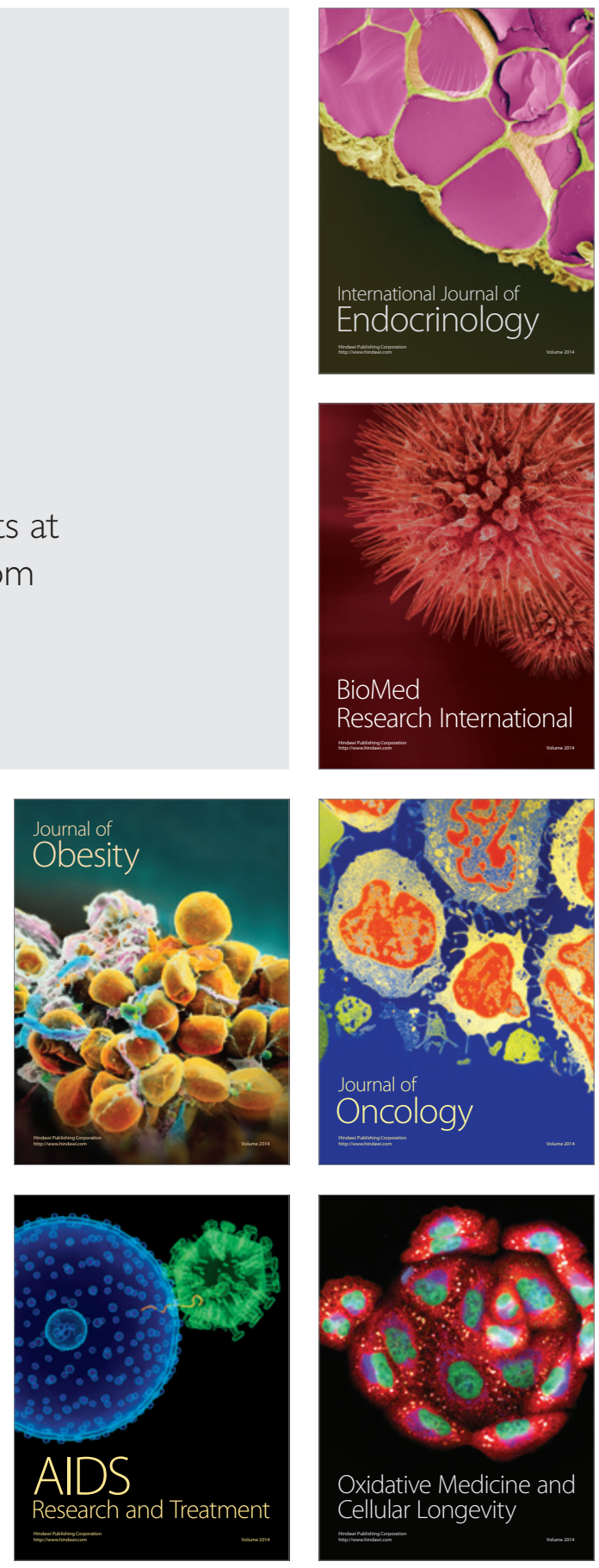